\title{
Structural health monitoring for bolt loosening via a non-invasive vibro-haptics human-machine cooperative interface
}

\author{
Mahmut Pekedis ${ }^{1}$, David Mascerañas ${ }^{2}$, Gursoy Turan $^{3}$, Emre Ercan ${ }^{4}$, \\ Charles R Farrar ${ }^{2}$ and Hasan Yildiz ${ }^{1}$ \\ ${ }^{1}$ Ege University, Faculty of Engineering, Department of Mechanical Engineering, 35100, Bornova, Izmir, \\ Turkey \\ ${ }^{2}$ Los Alamos National Laboratory, The Engineering Institute, Los Alamos, NM 87545, USA \\ ${ }^{3}$ Izmir Institute of Technology, Faculty of Engineering, Department of Civil Engineering, Urla, Izmir, \\ 35430, Turkey \\ ${ }^{4}$ Ege University, Faculty of Engineering, Department of Civil Engineering, 35100, Bornova, Izmir, Turkey \\ E-mail: mahmut.pekedis@ege.edu.tr
}

Received 15 November 2014, revised 5 May 2015

Accepted for publication 4 June 2015

Published 7 July 2015

\begin{abstract}
For the last two decades, developments in damage detection algorithms have greatly increased the potential for autonomous decisions about structural health. However, we are still struggling to build autonomous tools that can match the ability of a human to detect and localize the quantity of damage in structures. Therefore, there is a growing interest in merging the computational and cognitive concepts to improve the solution of structural health monitoring (SHM). The main object of this research is to apply the human-machine cooperative approach on a tower structure to detect damage. The cooperation approach includes haptic tools to create an appropriate collaboration between SHM sensor networks, statistical compression techniques and humans. Damage simulation in the structure is conducted by releasing some of the bolt loads. Accelerometers are bonded to various locations of the tower members to acquire the dynamic response of the structure. The obtained accelerometer results are encoded in three different ways to represent them as a haptic stimulus for the human subjects. Then, the participants are subjected to each of these stimuli to detect the bolt loosened damage in the tower. Results obtained from the human-machine cooperation demonstrate that the human subjects were able to recognize the damage with an accuracy of $88 \pm 20.21 \%$ and response time of $5.87 \pm 2.33 \mathrm{~s}$. As a result, it is concluded that the currently developed human-machine cooperation SHM may provide a useful framework to interact with abstract entities such as data from a sensor network.
\end{abstract}

Keywords: structural health monitoring, damage diagnosis, damage sensation, haptics, vibrotactile, sensory substitution, human-machine interface

(Some figures may appear in colour only in the online journal)

\section{Introduction}

In order to improve the reliability, safety and operational life of a structure, it is vital to monitor its integrity. The process of implementing a damage detection strategy for aerospace and civil and mechanical engineering infrastructures is referred to as structural health monitoring (SHM). Here, the damage is defined as changes to the material and/or geometric properties of these systems including changes in boundary conditions (Farrar and Worden 2013). For years, the research into SHM in particular has been more or less influenced by bioinspiration or biomimetics. There is a strong analogy between SHM and human sensing systems, and this relation is a real source of innovation. In other words, the SHM community has 
worked on structures that were equipped with various types of sensors having a nervous system similar to that of living skin organisms. Recently, bio-inspiration has been adapted to improve the performance of complicated sensor networks for SHM. In addition to that, as these sensor networks evolve, they become even more complicated with an increasing number of sensors (Loh et al 2015). There have been efforts using bio-inspired concepts to improve the SHM (Loh and Azhari 2012, Salowitz et al 2013). A prime example of a bioinspired SHM sensor was developed by Zhu et al (2010). Loh et al (2008) demonstrated strain sensing using layer by-layer thin films assembled with single-walled carbon nanotubes (SWNTs) and various polyelectrolyte species. SWNT-based films inspired by biological skin were also embedded in fiberreinforced composites for damage detection (Loyolo et al 2010). However, despite the advances in skin-like thin films, strain sensing can only be carried out in an averaged sense, in other words averaged over the area of the film. Currently, structural damage such as corrosions or cracks may not be detected using an averaged strain measurement. Strain sensing, as well as monitoring delamination and micro-crack damage were also studied by Böger et al (2008), and Nofar et al (2009) using bio-inspiration analogy. However, this analogy has remained superficial, because living tissues are really auto-adaptive smart structures that can control their integrity. At the micro-scale, the number of sensors in living tissues is incomparable to the nervous sensor systems of manmade structures (Balageas et al 2006). It was reported that there are 640000 sensory receptors to sense and monitor the changes in temperature, deformation, flow and injury damage in the entire system of the human skin (Schmidth 1986). Another analogy is often used comparing the nervous system of living tissues and structures integrated by sensors and equipped with a central processor (Beral and Speckmann 2003). The gap between living systems and artificial structures is smaller in this case and the study of the functioning of the nervous system and the brain is important when conceiving control systems. After the signals have been detected by the sensors embedded in the structure, a central processor can build a diagnosis or prognosis and predict the action to undertake. This process is often carried out with an SHM algorithm to remove the human factor from the SHM loop and build a system that is completely autonomous. However, a significant regulatory problem should be overcome before a fully automated SHM is applied to the structures. Currently, SHM analysis techniques do not have the capability to deal with this kind of problem. However, the human nervous system can easily adapt to a wide variety of variables such as pressure, singular force, strain and displacement. In other words, we are still struggling to build autonomous tools that can match the ability of a human to detect and localize a quantity of damage in structures. Therefore, there is a need for merging machine computational processes with human cognitive processes to improve the solution of SHM.

The concept of the human-machine SHM approach was recently demonstrated in a series of scientific conferences by the authors, before it was tested on human participants
(Brown et al 2013, Mascarenas et al 2013, Block et al 2014). Then, the approach's sensitivity was investigated on human participants using a three-mass-storey structure as a first experiment (Mascareñas et al 2014). As outlined in those studies, generally the approach was inspired by the neuroscience community. The idea of neural plasticity, which is a branch of neural science, has been explored for a number of years. Neural plasticity refers to a multitude of different processes of reorganization within the brain, each of which affects the way information is processed and may ultimately result in behavioral changes (Grosse-Wentrup et al 2011). Sensory substitution, which is used in neural plasticity, refers to the translation of sensory information that is normally available via one sense to another. It has been demonstrated in various studies that sensory substitution is technically and practically possible (Melchner et al 2000, Sharma et al 2000, Visell 2009). It can happen through all sensory systems, such as touch-to-sight, or within a sensory system such as touch-totouch. Paul Bach-y-Rita initiated a project to explore late brain plasticity, which soon after was to grow into a wide area of sensory substitution studies (Bach-y-Rita and Stephen 2003). The best example is the phenomenon of 'blindsight' by visually impaired people through which the heightened use of auditory channels enables individuals to discern objects, obstacles or structures in front of them (Weiskroniz 1986). The studies mentioned above suggest that humans could achieve a new sense over time. The other application of neural plasticity is echolocation. It was outlined in some studies that blind persons can discern their surroundings by producing clicks with their mouths and listening to the returning echoes, in a manner similar to bats (Stoffren and Pittenger 1995). The techniques used in echolocation suggest that humans could achieve new senses for abstract data. This approach could combine ultrasonic-based SHM measurements for future studies using the echolocation concept. By outfitting humans with the vibrotactile interface, they may localize ultrasonic scatterers that are representative of damage in a structure.

Recent advancements in SHM are towards the application of statistical signal processing techniques to diagnose the damage in structures. Such methods are based on signatures obtained from the sensor data to extract the features that change with damage. These features can then be discriminated in a pattern classification framework. A statistical pattern recognition involves the following processes: (1) operational evaluation, (2) data acquisition, (3) feature extraction and data reduction and (4) statistical model development (Sohn and Farrar 2001, Cheung et al 2008). In this study, the human neural network (NN) capability of damage localization based on vibrohaptic actuators is explored instead using statistical model development. The reason for using the human $\mathrm{NN}$ comes from some studies that show humans have the capability of classification. Moreover, some studies show that humans often exceed the contemporary algorithms (Schwamb et al 2012). Stallkamp et al, presented a comparison of the traffic sign recognition performance of state-of-the-art machine learning algorithms and humans in detail. They found that humans were able to 
recognize types of traffic signs in such conditions (Stallkamp et al 2012). One of the most famous pattern recognition algorithm artificial neural networks (ANNs) was inspired by the high performance of the human brain, which, with its sophisticated topology, can determine and extract significant features for feature extraction problems, so it is intuitively reasonable to simulate a similar structure for the feature extraction problem (Dashti et al 2010). Vapnik revealed the advantage of the inclusion of 'empirical' concepts and opened the way for machine learning to take advantage of human factors beyond conventional ANN architecture (Vapnik 1998). Barlow (1969) made seminal contributions to the role of sparse coding information in the brain's performance. It is known that machines are assumed to be good for computational processes, whereas humans are best for facing unforeseen or unknown conditions (Bainbridge 1987). The 'human-out-of-loop' mechanism was often stressed (Hoc 2000). According to the study of Vanderhaegen, that is probably one of the reasons why human air-traffic controllers rejected an automatic mode of conflict allocation between human and machine (Vonderhaegen et al 1994). The studies listed above are a part of the research that depicts the human cognitive effect on computational studies. In this study, we think that by merging the human cognitive system with the machine computational process, a higher computational process could be built for SHM problems. In other words, that the formula of 'human + machine' will result in a better process for enhanced performance is one of the main ideas behind the concept of cooperative SHM. Therefore, the cooperative SHM concept appeared: the combination of the idea of high computational performance of humans and machines teaming with the idea of sensory substitution. In the future, perhaps it could be considered to interact the highly developed human nervous system distributed measurement system to solve the SHM problem better. In the cooperative SHM approach, the data collected by a distributed SHM measurement system are interfaced with a human nervous system using the principle of sensory substitution. The current trends in SHM are to obtain the sensor network data from the structure, process them with various tools and present them with visual graphs. Here, instead of presenting the results of SHM visually, they are represented to the human using noninvasive haptic vibrations. We anticipate that this approach has potential applications where humans are in the loop such as remotely piloted vehicles (aircraft or watercraft etc), teleoperated systems and robots. For instance, to the best of our knowledge the possibility of interfacing the SHM system onboard for robotic platforms has been largely unexplored to date. However, if the data of robotic SHM system are outfitted with the human nervous system, this can help robots to avoid dangerous stimuli in their environments. Moreover, in the case of semi-autonomous or teleoperated robots, it would be helpful if an embedded SHM system onboard a robot could be elegantly interfaced with the nervous system of the human controlling it. This would enable the human operator to avoid commanding actions that could potentially cause damage to the robot.
The layout of the study is as follows. First, the methodological concept of the human-machine SHM cooperation concept is summarized and the experimental setup of the structure is demonstrated. Next, hardware and software development for the proposed experiment is presented. Principal component analysis (PCA) modeling of the time signals is explained. Then, the types of stimuli presented to the human subjects with haptic experimental devices are expressed. Finally, the results obtained from human subject tests are discussed.

\section{The methodological concept of human-machine SHM cooperation approach}

The human-machine haptic SHM paradigm has been given in detail in previous work (Mascareñas et al 2014). In general, the cooperative SHM approach consists of the stages outlined in figure 1. The numbers depict the sequence data processes. Initially, a structure of interest is equipped with a distributed measurement system. These measurements could be accelerometer, strain, voltage or temperature. Next, the data are collected from the distributed measurement system. (1) The data are then normalized. (2) Multivariate statistical analysis is applied to reduce the data. (3) Once the data have been reduced with statistical techniques, the next step is to encode the data in a form that can be sent to a device. The encoded data are referred to as 'haptic code'. This is then used to modulate the actuation of a set of actuators such as haptic motors that are interacted with a human.

In this study, haptic actuators were used to stimulate the human nervous system. The nature of this haptic encoding depends on many factors including the type of haptic actuation used, the dynamic characteristics of the haptic actuators, the location on the body where the haptic actuation is applied, the dynamic characteristics of the portion of the body the actuators are applied to, the number of available haptic actuators, the characteristics of the basis that can represent the pre-processed data in the most sparse fashion, and the representative spatial/temporal timescales of the data. Once the physical stimuli are applied to humans, the hypothesis at this point is that the human will develop a sense of the stateof-health of the structure in a manner similar to the phenomenon of 'sensor substitution' over time. The concept is that the human becomes aware of changes in the structure as a result of communication from the haptic interface. (4) Once the human results are acquired, their responses are evaluated statistically to figure out significant differences between human subjects and damage cases. (5) The eventual goal is to enable humans to make judgments on the performance of structures, and to decide what, if any, action should be taken. To conduct the flow steps given in figure 1, a software application for the admin coordinator and human subject was developed using the PYTHON object oriented programming language. During the experiment, the software allowed us to: (i) send signals to the haptic experimental device to stimulate humans, (ii) supply feedback for the participants, (iii) log and save the responses of the subjects for different cases. 


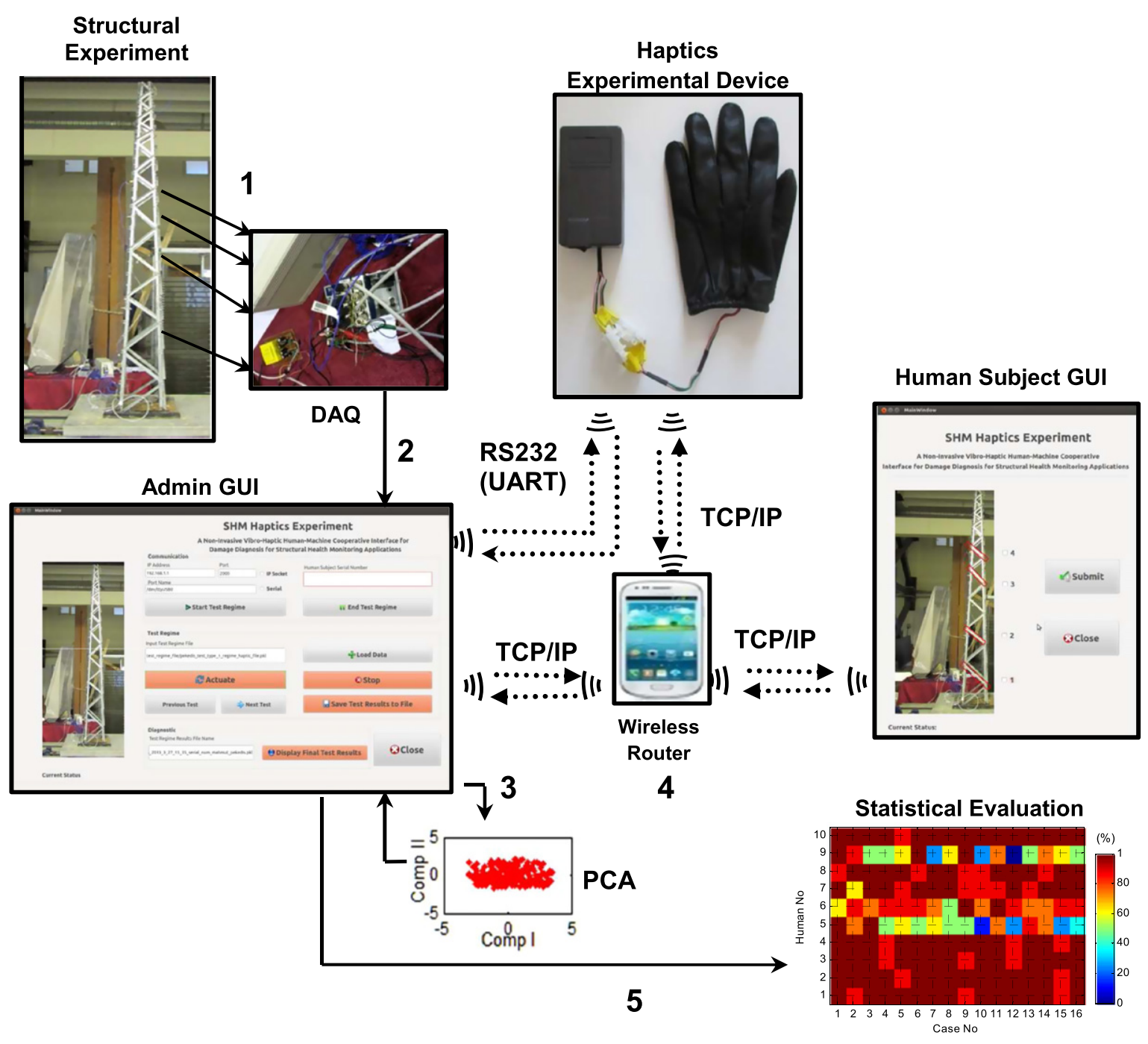

Figure 1. Flow chart of the developed human-machine cooperative SHM system.

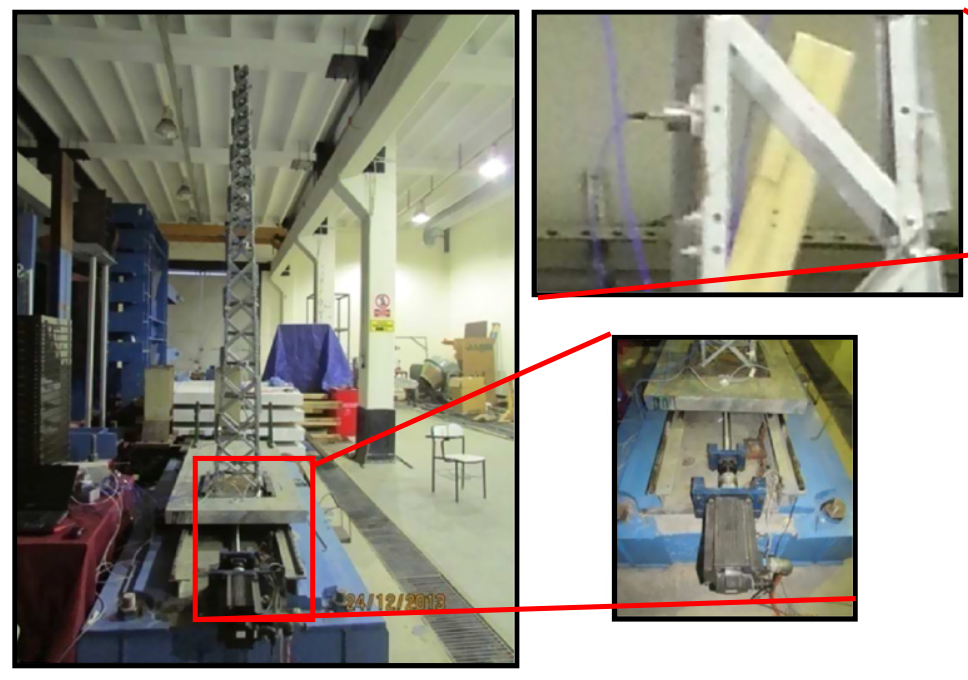

Lateral view

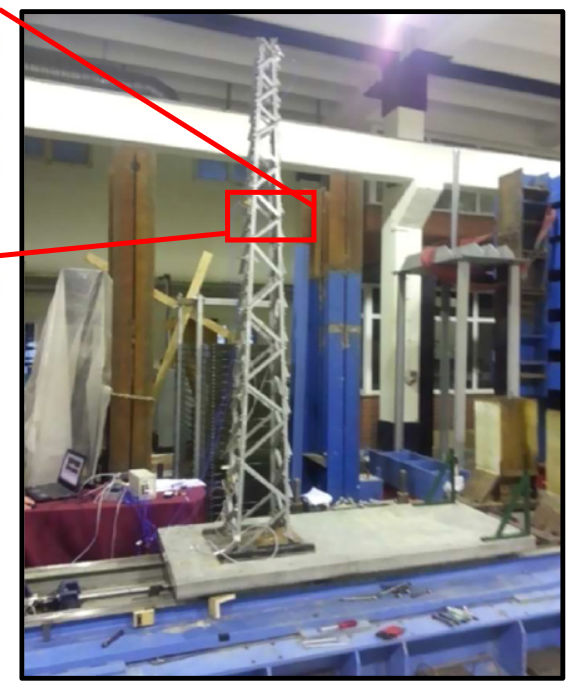

Front view

Figure 2. Experimental setup of the tower structure. 


\subsection{Experimental setup}

In this study, a steel tower structure having bolted lattices was manufactured as a test platform as shown in figure 2. This structure was mounted on an aluminum plate that was located on horizontal rails and excited with a servo motor (Omron) that vibrated at a single frequency. The structure that was located on the plate slid on rails that allowed movement only in the lateral direction. The steel lattice tower consisted of four legs. The joints were designed to transfer the axial forces safely and also provide adequate strength and ductility to resist fracture. The structure, manufactured from low carbon steel, had a height of $2.95 \mathrm{~m}$ while the span of the base was $300 \mathrm{~mm}$. The dimensions of the members were L $20 \times 20 \times 2$. The position of the bolts could be set to vary the extent of impacting that happens at a special excitation value. In this experiment, the bolt tip was set to be $2 \mathrm{~mm}$ away from the member. By varying the number and location of loosened joints, the structure could have a wide variety of damage scenarios. The structure was placed on a shake table and excited by a servo motor. The table slid on rails, which were mounted on motionless supports on two sides of the table. The desired motion was transmitted to the moving table by means of the servo motor, an infinite screw and a bolted connection. The entire system was placed on a high stiffness reinforced concrete foundation. This foundation minimized extraneous sources of unmeasured excitation from being introduced through the base system. Four accelerometers (PCB, Piezotronics) with nominal sensitivity of $1000 \mathrm{mV} \mathrm{g}^{-1}$ were bonded to the center line of the tower members at the front side of excitation direction to measure the dynamic response of the system. The heights of the accelerometers from bottom to top were $85 \mathrm{~cm}, 166 \mathrm{~cm}, 198 \mathrm{~cm}$ and $295 \mathrm{~cm}$, respectively.

The location of the servo motor was centered at the edge of the table. The tower and accelerometers were placed on the same line as the shake table's lateral axis to minimize the torsion effects of excitation of the system. NI 9234 type data acquisition was used to get the data. Labview software was used to process the data. The analog accelerometer sensors were discretized with 4268 data points sampled at 1707.06 Hz. Exciting the structure while no joints were loosened produced a linear dynamic response of the structure, while loosened joints or any combination of joints resulted in a non-linear dynamic response. The sample parameter yields were $2.5002 \mathrm{~s}$ in terms of duration. Initially, the natural frequencies of the system were determined. The natural frequencies of the base system model (all bolts fixed) were found by using an earthquake simulator to create an impulse at the base of the tower. The shake table was driven by a velocity of $600 \mathrm{~mm} \mathrm{~s}^{-1}$ for $1 \mathrm{~s}$ and then it was stopped. The resulting structural free vibrational responses were recorded. A simple Fourier transformation yielded the system's natural frequencies as 13.33, 13.74 and $14 \mathrm{~Hz}$ for the first three modes. Then, the excitation level was set to a $14 \mathrm{~Hz}$ sinusoidal wave for the third mode. This excitation signal was selected to prevent rigid body motions of the structure. Measurement sensor data were categorized according to
Table 1. Various scenarios of the structural conditions (1 depicts the bolt is loosened, while 0 depicts the bolt is not loosened).

\begin{tabular}{llllll}
\hline & & \multicolumn{4}{l}{ Bolt loose location representation } \\
\cline { 3 - 6 } Case & $\begin{array}{l}\text { Number } \\
\text { of tests }\end{array}$ & Region 1 & Region 2 & Region 3 & Region 4 \\
\hline 1 & 8 & 0 & 0 & 0 & 0 \\
2 & 8 & 1 & 0 & 0 & 0 \\
3 & 8 & 0 & 1 & 0 & 0 \\
4 & 8 & 0 & 0 & 1 & 0 \\
5 & 8 & 0 & 0 & 0 & 1 \\
6 & 8 & 0 & 0 & 1 & 1 \\
7 & 8 & 0 & 1 & 0 & 1 \\
8 & 8 & 1 & 0 & 0 & 1 \\
9 & 8 & 1 & 1 & 0 & 0 \\
10 & 8 & 1 & 0 & 1 & 0 \\
11 & 8 & 0 & 1 & 1 & 0 \\
12 & 8 & 1 & 0 & 1 & 1 \\
13 & 8 & 0 & 1 & 1 & 1 \\
14 & 8 & 1 & 1 & 1 & 0 \\
15 & 8 & 1 & 1 & 0 & 1 \\
16 & 8 & 1 & 1 & 1 & 1 \\
\hline
\end{tabular}

whether bolts were loosened or not. Sixteen different damage scenario combinations $\left(2^{4}=16\right)$ were simulated. The acceleration time series for the sixteen different scenarios were collected as given in table 1. Eight measurements were obtained for each scenario, and a total of 128 test measurements were collected.

\subsection{Hardware design}

An electronic board based on a microcontroller was developed to actuate the vibratory haptics elements. The architecture of the actuator control system was based on an embedded code with a microcontroller unit to deliver the signals to the actuator. An Arduino Mega 2560 based on ATmega2560 was used for the human-machine prototype. The prototype for the developed SHM human-machine interface is given in figure 3 . The prototype device comprised four miniature haptics elements (Precision Microdrives, London, UK), each independently providing identical force amplitude. The diameter, height and mass of the vibratory element were $10 \mathrm{~mm}, 3.4 \mathrm{~mm}$ and $1.2 \mathrm{~g}$, respectively. Each of these haptics elements was controlled with a TIP 120 Darlington transistor and driven using pulse with modulation (PWM) that was generated by the microcontroller. The operating range of these vibratory elements was $2.2-3.8 \mathrm{~V}$. Here, the driving current increased the vibration frequency and hence the produced force amplitude was proportional to the PWM duty cycle. These vibratory actuators were attached between the two cloth layers of a glove. They were attached between the two layers of the glove for the finger joints (Art. interphalangealis proximalis ligament) to capitalize on pacinian corpuscles. The vibrotactile actuators were situated on the back of the left hand. The back of the hand may be advantageous since it has been found that vibrations are best on hairy bones (Bear et al 2007, Myle and Binseel 2007). In 


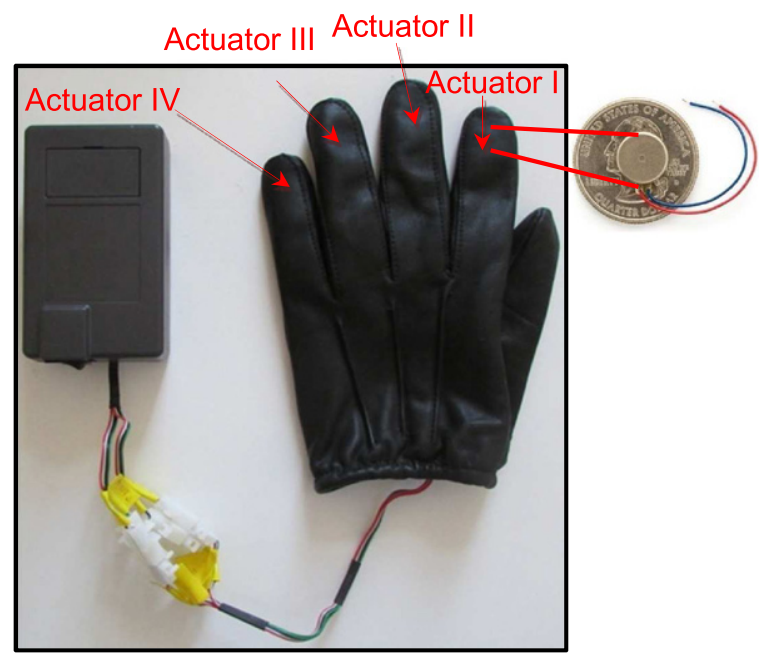

Figure 3. Haptics experimental device (glove).

addition to that, according to the law of mobility, the closer the location to anatomical landmarks (joints like wrist or elbow), the better the localization of stimuli. Therefore, humans can localize the same vibration better at a position where movement is allowed.

The haptic codes were sent by a laptop computer through an RS232 serial interface with an X-bee dongle and received by the X-bee radio receiver to transmit them to the microcontroller. All data transmitted to the microcontroller encoded the response of one of the four actuators presented in the haptic interface. This allowed us to set the frequency, the timbre and the duration of stimulation of each vibrotactile element. By mapping the location of each actuator on the interface, signals could be tailored to align groups of actuators in tandem by creating sweeping, alternating, circling or more complex patterns. In this study, the generated actuation patterns were given in detail at the part of stimuli presentation. A resistance with a value of $1 \mathrm{k} \Omega$ was connected to the base component of the transistor, and a rectifier diode with a value of $1 \mathrm{~A}$ was connected to the components of each vibration actuator. The microcontroller was programmed to represent the digital codes that were transferred from the computer to the haptic prototype wirelessly as a vibration pattern for each stimulation.

Figure 4 shows the measured current at different dutycycles. As can be seen, the relation between the input dutycycle and the current is linear. As the PWM duty cycle increases, the vibration magnitude of a vibratory element increases. Patterns representing the structural data are encoded and sent wirelessly to the prototype microcontroller that is worn by the user. Here, once the signals are received by the microcontroller, an embedded program distributes the signals to the actuators to initiate the vibrators. The microcontroller, enclosure, radio receiver, circuit board, actuators and power unit are assembled so as to be a completely portable mobile unit.

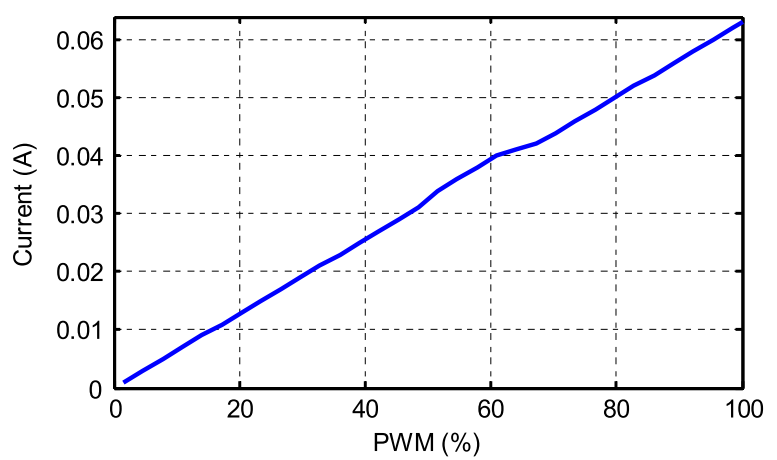

Figure 4. PWM duty cycle versus current delivered by the microcontroller.

\subsection{Software design}

To test the human-machine interface SHM concept, a graphical user interface (GUI) was designed for the administrator and human subject using a PyQT4 development suite for creating GUIs in PYTHON on a Linux Ubuntu 12.04 operating system. Figures 5 and 6 represent the developed GUIs for cooperative SHM facilities for admin and human subject respectively. The admin GUI is capable of analyzing the acquired data from sensors, generating haptic data, shuffling and sending them both to the human subject GUI on TCP-IP to the developed prototype via a serial or socket port connection simultaneously, receiving the human responses and measuring human response duration. The network connection between the admin GUI, human subject GUI and haptic experimental device is given in figure 1. Here, a Samsung Galaxy SIII mobile phone was used as a WiFi hotspot to provide the connection between the admin and human subject. The admin connects to the human via TCP-IP with a robotic operating system (ROS) to send the questions to the human (publisher) and to acquire the human response (subscriber). The codes are transmitted at the same time to both the human GUI and the haptic prototype.

In the admin GUI, first the connection type should be chosen for transferring the haptic codes from the admin GUI to the developed experimental haptic device. If the haptic codes are transmitted on an IP socket, an XBee Wi-Fi type product is used with the haptic device, and input widget of 'IP address' and 'Port' number for the X-Bee should be filled, then the 'IP Socket' radio button is signed. If the haptic codes are transmitted on serial communication, an XBee ZigBeemesh based on an RF type product should be used with the haptic device, and an input widget of serial 'Port Name' for the X-Bee dongle, which is connected to the computer, should be filled, then the IP Socket radio button is checked. In this study, a wireless XBee mesh type product based on RS232 serial communication was used. The 'Stop' button stops sending signals that are transmitting on the IP-socket or serial port from the admin GUI to the haptic device. The 'Load Data', allows the user to load the test regime file. The 'Actuate' button allows the user to transmit the haptic codes to both the human subject GUI with TCP-IP type connection 


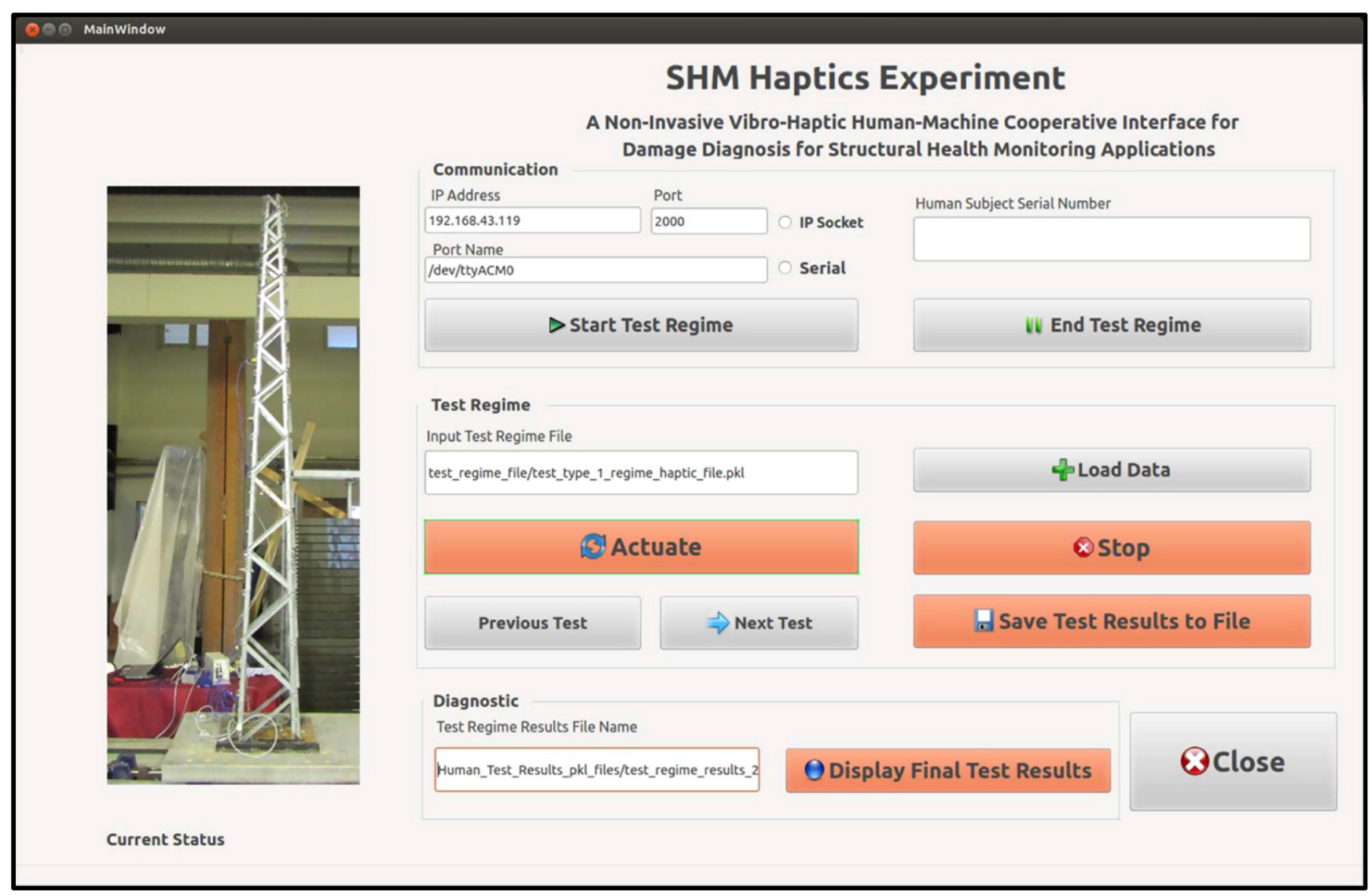

Figure 5. Graphical user interface (GUI) for administration.

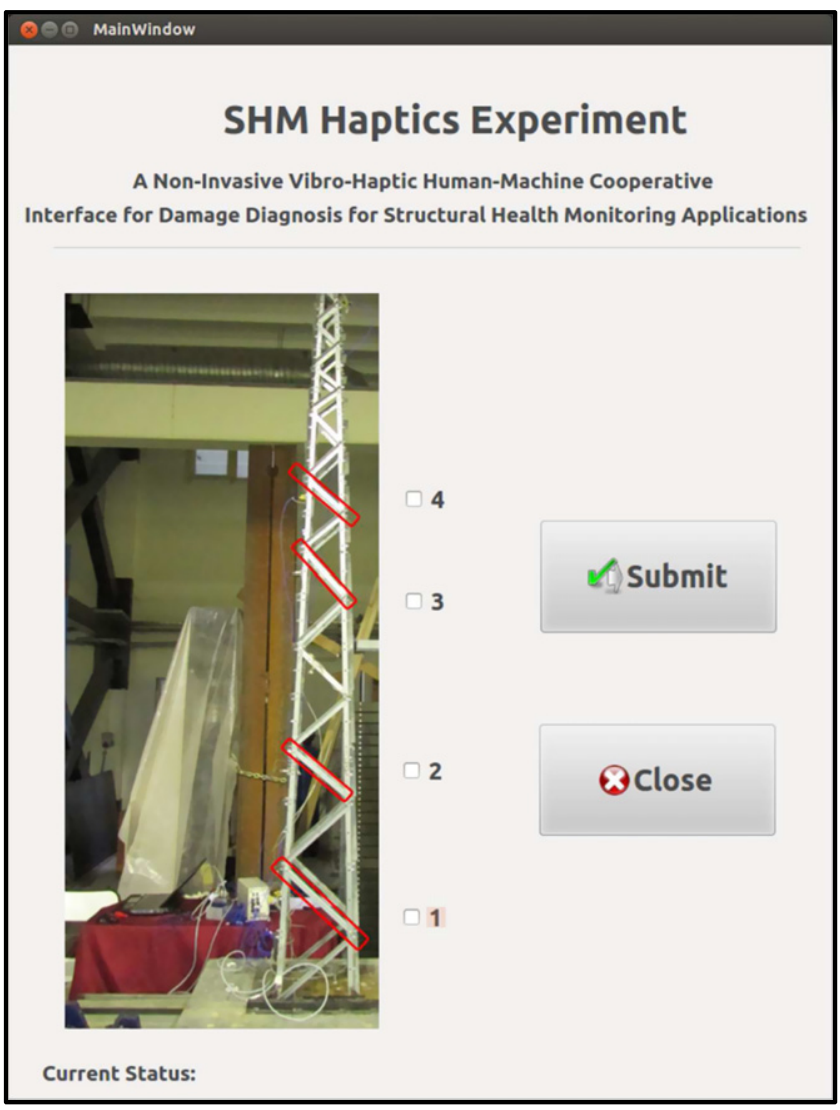

Figure 6. Graphical user interface (GUI) for a human subject. and to the haptic device via the serial port using the wireless $\mathrm{X}$-Bee radio.

In the human subject GUI, check boxes allow the user to choose the answers that reflect which bolt/bolts are loosened. After the human subjects send their responses to the admin at each test, the clicked checked boxes are automatically removed, which allows the facility to be used for the next test. The 'Submit' button allows the user to send his/her responses to the admin GUI after clicking the check boxes.

The admin and human subject communicate via GUIs to follow and monitor the test status with the line 'Current Status'. For example, when the admin sends the haptic codes to the human subject, 'test_index = test_no of 128 is actuated' is shown in this line as well as when the human subjects receives the message 'Message received for shuffled test index $=$ shuffled_test_number' in the human interface. When the human subject sends his/her responses, 'Message is sent' is shown in this line as well as when the admin receives the message 'RESPONSE RECEIVED! test_index = test_no of 128'. It should be noted that test_no and shuffled_test_number are integer values that show the current test number and shuffled test number, respectively in the admin GUI. In addition, the digital haptic codes that are sent to the haptic experimental device by the admin GUI are transferred back and can be monitored in a terminal in order to verify that the represented haptic stimuli on human subjects work 
correctly. This process was conducted by programming the microcontroller unit.

\subsection{Procedure}

The testing procedure begins with data collection, storage and categorization of data collected from the test structure. For consistency, data used in testing will be pre-recorded, and the same set of data must always be used to indicate a given setup. Data are categorized according to which bolt/bolts are or are not loosened as given in table 1 . For each of the sixteen combinations in structure, a data structure containing the recorded accelerometer data is maintained. Each of these data structures constitutes an instance of a 'test'. Tests are combined to create one 'test regime'. In this research, three test regimes for each stimulation type containing all 16 cases and totaling 128 tests were used, though in further research more test regimes could be created, i.e., where the number of times one test occurs in the regime could vary. Once the test regime has been created for each stimulation type, the test could be applied on humans. Initially, a poster representing the concept and how the system works is shown to each subject. Then, the subject's test session begins with familiarizing the human subject with the interface and the actuation patterns that may be encountered due to various structure responses. This step is referred to as training. Subjects are outfitted with the glove interface and allowed to make adjustments until comfortable. Once comfortable, a test regime is loaded into the software. For each test, the subject is informed what combination of bolt loosening is being used, and the corresponding test is used to actuate the glove. The full test regime including 128 tests is used, exposing the subject's ability to internalize the sensations caused by bolt loosening. Training is completed when the subject indicates that he/she feels ready. After completion of testing, the process begins to determine how well the subject has learned to feel the structure health. Using developed interfaces (one for each test) between the subject and the test administrator, the vibrohaptic interface allows the subject to input his or her diagnosis of which bolt is loose. Data are recorded indicating which bolt they believe to be loose, the amount of time it takes the human subject to make a decision regarding which bolts are loose, and the number of repeats of any test case that they request. Then, these data are used to begin characterizing the accuracy and certainty of the human subjects.

\subsection{Participants}

The experiment conducted in this research was approved by the Ege University Medical Faculty ethics committee with document 13-11/104 prior to the study. The subjects used in the experiment were mainly research assistants and students graduated from Ege University. All subjects were between the ages of 25 and 34 . The mean and standard deviations of the human subject ages were 29.6 and 4.61, respectively. The human subjects were encouraged to adopt a comfortable position that allowed them to feel vibrotactile stimulation better. All of the subjects wore the glove on their left hand

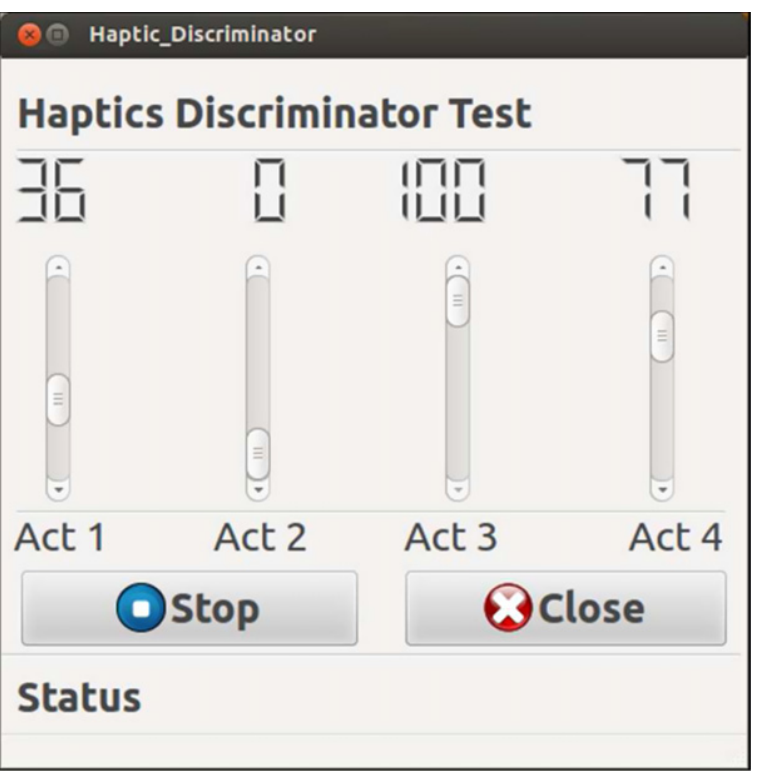

Figure 7. Graphical user interface GUI for haptics discriminator test.

and used the mouse with their right hand to choose which bolts they believed to be loosened. They were seated at a desk with a human subject software screen.

First, a discriminator test was performed on the participants to determine whether the participants had the capability to discriminate the four tactips from each other before the experimental session. This test consisted of a verbal response indicating whether the participants could identify and discriminate the vibration magnitude of each tactip in the discriminator stimulation. The developed GUI for the discriminator test is given in figure 7. The sliding button was scaled with a 0-100 PWM duty cycle. Here, none of the subjects reported any difficulties in discriminating the haptics actuators from each other. After completing the discriminator test for each subject, the goal was to test the SHM concept for the structure on the same human subjects. Next, three different vibrotactile stimulations as explained in the stimuli section were applied to the humans with the cooperative SHM experimental glove device. Each of the vibrotactile stimulations consisted of two sessions. In the first session, the subjects were allowed to familiarize themselves with the pattern of stimulation at training. All of the human subjects were told how the system works and the structural health (bolt loose/no bolt loose) for each test was explained at this training period. Upon completion of testing, the first step was to determine how well the subject had learned to feel the structure.

\subsection{Principal component analysis}

PCA is a multivariate analysis technique that was first presented by Pearson (1901). The goals of PCA are to extract the most important details from the data, compress the size of the dataset by keeping only the important information, and to analyze the structure of the observations and variables. In order to achieve these goals, PCA calculates new variables named principal components that are linear combinations of 
the original variables. The first principal component is required to have the largest possible variance. The second principal component is calculated under the limitation of being orthogonal to the first component. The other components are calculated in the same way. The new obtained variables extracted from PCA are called factor scores; these factors can be calculated by the projection of the variables onto the principal components. In order to build a PCA model, it is required to arrange the data obtained from the experimental sensor.

The implementation of the PCA technique in SHM steps is as follows.

(a) Organize the measurement data set as an $I \times J$ matrix, where $I$ represents the measured variables and $J$ represents the experimental trails.

(b) Standardize the data

(c) Calculate the eigenvectors and eigenvalues.

(d) Choose the first and the second eigenvectors as the principal components.

(e) Transform the data into the first and second principal components.

(f) Calculate the loadings for the first and second principal components.

(g) Normalize the loadings in a range of $0-1$, then scale them for the PWM duty cycle in a range of 0-100, and finally encode them as haptic stimulation codes to transmit them from the administrator GUI to the haptic experimental device. Normalized loadings of the first and second components are given in figure 8 . The sequence numbers from 1 to 8 represent the first two components of the variables. In other words, 1 and 2 represent the loadings of the first and second components of the first channel, 3 and 4 represent the loadings of the first and second components of the second channel, 5 and 6 reflect the loadings of the first two components of third channel, and the last two numbers depict the loadings of the first two components of the fourth channel.

\subsection{Stimuli presentation}

The sense of touch can be perceived through a combination of many sensory pathways. Sensory signals through human hands are interpreted as temperature, texture, location, size, movement, and, in some situations, pain. For tactile perception in glabrous skin (i.e., hairless skin such as the palms of the hands), receptors are embedded within the skin and are referred to by receptor ending, speed of action, or psychophysical channel. The exact frequency range of sensitivity of each channel is not well defined, partly due to the difficulty of stimulating any one channel in isolation and also due to individual differences (Mansfield 2005). Therefore, many related efforts should be focused on characterizing the human perception of haptic stimuli parameters to determine what makes the stimuli distinguishable. It would be desirable to investigate the human ability to subjectively recognize the haptic stimuli that might provide an opportunity for SHM applications.

This study is multi-disciplinary in the sense that we are trying to learn how results from the psychophysics procedures can be applied to SHM. Many relevant psychophysical properties are known for human sensation. These include the relevant ranges of frequency sensitivity, receptor density, receptor field size and sensory correlations: all quantities that are highly salient. The most frequently cited is the two-point threshold. The two-point thresholds have been directly correlated to the skin sensitivity level. The method of limits is one of the most frequently used techniques for determining sensory thresholds, and although it is considered to be less precise than the method of constant stimuli, it is less time consuming, resulting in being considered to be more efficient (Gescheider 1997). The perception of vibration across most of the frequency continuum $(10-300 \mathrm{~Hz})$ is mediated largely by two psychophysical channels, the pacinian and nonpacinian (Verrillo et al 1969). The nonpacinian channel II, has a sensitivity over much of the $10-300 \mathrm{~Hz}$ frequency range that is only slightly less than that of the nonpacinian I channel. The two main features of a vibrotactile stimulus are vibration amplitude and frequency.

The perception of various amplitudes and frequencies on different body parts has been studied in depth. The ability to discriminate changes in frequency depends on the given stimulation frequency (Verrillo 1985). The amplitude discrimination threshold (the lowest perceptible difference between the two signals) for human hairy skin is known to be approximately an order of magnitude higher than for glabrous skin (Merzenich and Harrington 1969). The thresholds depend on the reference stimulus, and varies with stimulation frequency. For hairy skin, it decreases at higher frequency. The best result obtained for the ability to discriminate changes was when the frequency was lower than about $200 \mathrm{~Hz}$ (Mahns et al 2006).

There are various methods to generate skin sensation. They can be categorized in various types like piezoelectric actuators, thermal feedback, voice coil motors and electrical pulses. A vibrating motor uses a counter-weight and a motor to vibration. These motor vibrations can be operated at low voltage $(1-5 \mathrm{~V})$. They have been used in research of sensory substitution for the design of prostheses (Dhillon and Horch 2005, Cipriani et al 2012), cyber (Cipriani et al 2012) and fluid hands (Christian et al 2006) due to their low cost and size. Another haptic stimulation approach is functional neuromuscular stimulation which provides stimulation directly to the neuromuscular system. Electrodes are placed in muscles and the neural system. Because neuromuscular stimulation is invasive and painful it is not a preferred method. In this study, it was aimed to capitalize on the sense of touch by targeting pacinian corpuscles in the hand.

The microcontroller unit (MCU) is designed to control the activation of the vibration tactips to create the haptic pattern from the haptic codes transmitted from administration. The temporal characteristic of the activation of each vibrohaptic actuator's duration of activation and time interval between actuations were arranged by coding the MCU, which 
Case 1

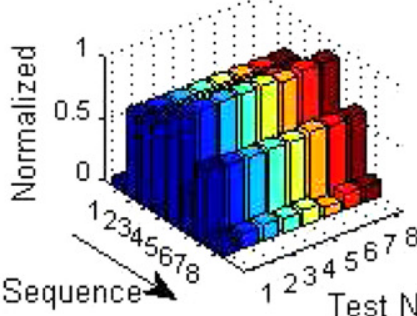

Case 5

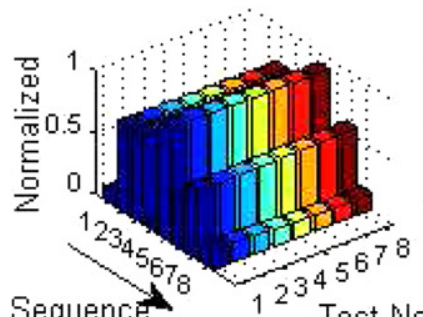

Case 9

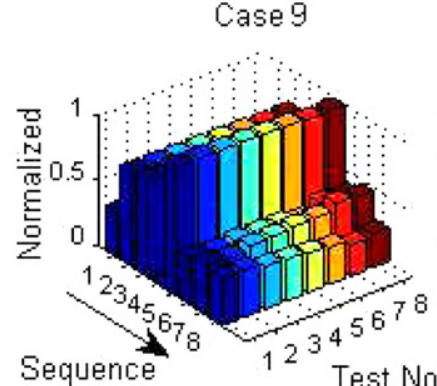

Case 13
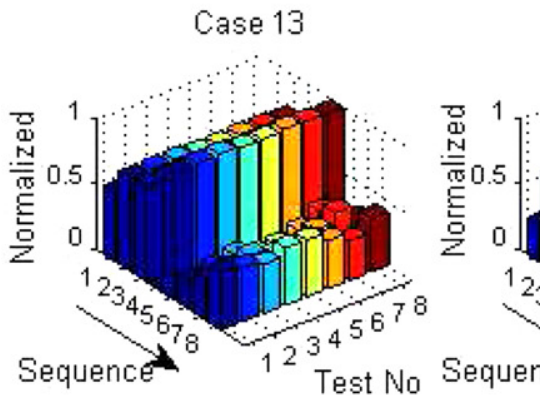

Case 14
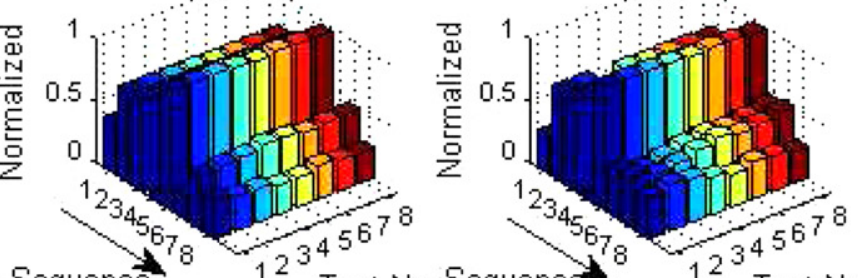

Case 7

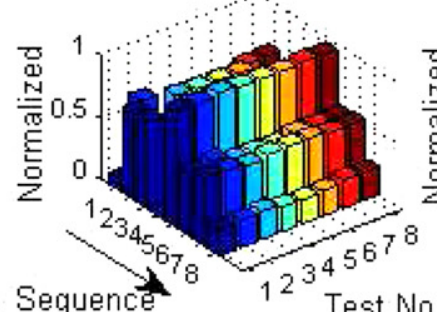

Test No

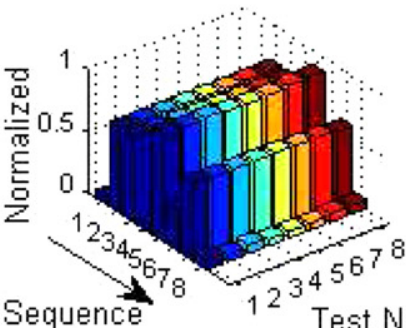

Case 6
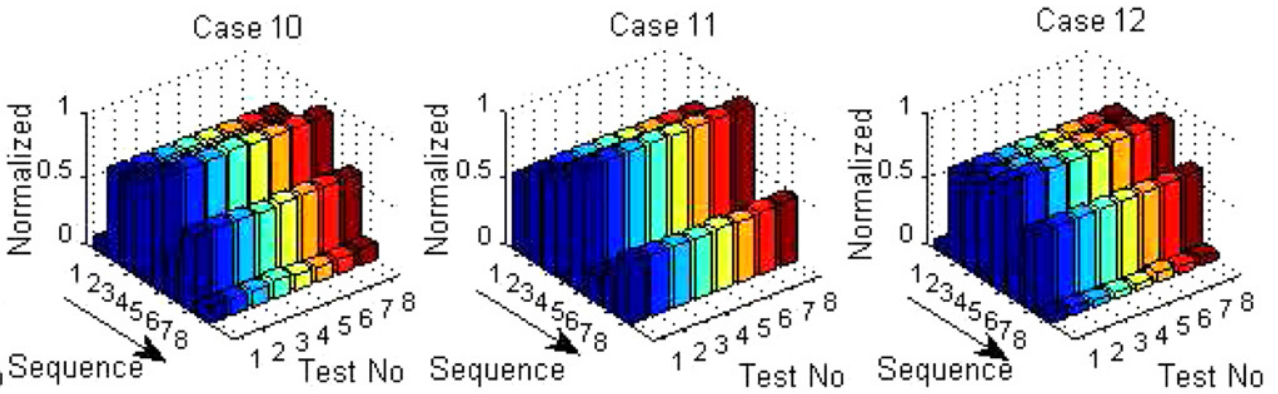

Case 4

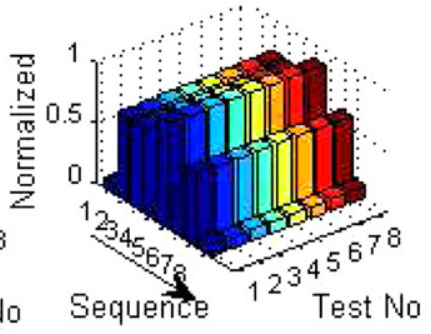

Case 8

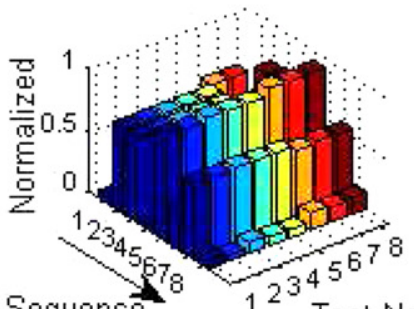

Test No
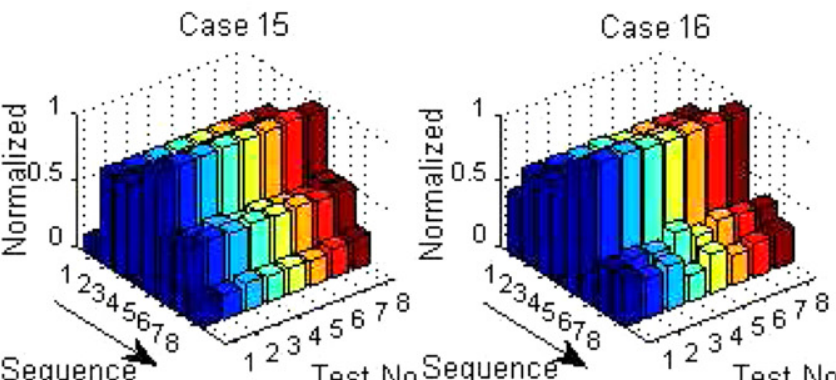

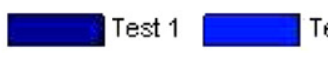

Test 2

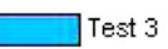

$\square$ Test 4

Figure 8. Normalized loadings of the first and second components.

transforms the haptic codes' array patterns into voltages to drive each of these haptic actuators. In this study, three different stimulations were performed for the human to recognize the damage in the structure. In some studies reported in the literature, humans appear to have the ability to recognize unique periodic haptic stimuli and their associated meanings. For example, Chan et al (2005) observed a 95\% successful recognition rate of a set of seven unique periodic haptic stimuli and their associated meanings, following a short learning period of three minutes. Additionally, Enriquez and MacLean (2008) observed that humans could successfully learn and recognize abstract meanings associated with individual parameters of periodic haptic stimuli, such as waveform and frequency. These studies showed the potential ability of humans to learn and remember abstract meanings associated with any haptic stimuli for extended periods of time. Based on this, we considered that human perception may recognize and distinguish the raw data obtained from the four sensors to characterize the damage in a structure, because the bolt loss during the excitation produces a nonlinear behavior that simulates the damages in the structure. The idea in the first stimulation is to investigate whether humans 
become aware or not of this damage using time series data of sensors. The pre-processing steps were also used in the study conducted by the authors for a three-mass storey structure (Mascareñas et al 2014). They investigated the human ability to discriminate between the eight damage classes. In the previous study, the damage was conducted through a bumper system, and a total of eight damage cases were investigated. Specifically, they implemented logarithmic data processing procedures to generate vibrotactile stimuli. They represented the participant's responses in a confusion matrix, and found the average score of participants to be $66.5 \%$. It is noted that, in a previous study, the data points of time series were preprocessed with decimating, shifting and dividing steps. More details can be found in Mascareñas et al (2014). In the current study, we explore the potential of humans to discriminate 16 structural cases. In order to make large measurements that were easier for humans to discriminate, they were reduced using the PCA. Note that this step is similar to pre-processes mostly conducted in machine learning algorithms. However, also note that the approach used here is not a statistically based classifier because the decision making about the structural state is conducted by a human. The details of implementing the PCA with sensor measurement to generate haptic stimuli are given in the previous section.

In the first stimulation we aimed to figure out the human capability for damage localization in the tower structure. It was noted that in the first stimulation, we did not benefit from the advantages of the machine computational process (the machine refers to the computer). In other words, no signal processing or classification techniques were performed on signals during the creation of the stimulation. Only human intelligence was used in the first stimulation. The basic implementation of the first stimulation procedure is as follows.

Initially, time points from each signal were obtained in a range of 0-585.94 ms. The mean frequency of the obtained signals was $14 \pm 0.03 \mathrm{~Hz}$. Since it is difficult for the human to remember the wave shape of the signal from these time points with haptic stimuli in a range of $0-585.94 \mathrm{~ms}$, the frequency of all the signals was reduced by a factor of 4.4373 . This process reduced the initial sampling rate of these signals to the lower rate. The final time range of all signals was increased from $585.94 \mathrm{~s}$ to $2600 \mathrm{~ms}$ by performing this reduction process. Next, the signals consisting of the modified time points were scaled in a range of 0-100 to represent them with the PWM duty cycle. Finally, all the signals were mapped onto a vibration motor according to their arrangement number to stimulate the human hand. (Channels 1, 2, 3, and 4 were mapped onto vibration motor numbers 1, 2, 3, and 4, respectively as given in figure 9). The duration for each signal shape actuation and the pause between them was arranged to be $2.6 \mathrm{~s}$ and $1.3 \mathrm{~s}$, respectively (similar to the study of Morley and Rowe 1990, Cipriani et al 2012). At the end of completion of the fourth vibration motor stimulation, the vibration motors restarted stimulating in an order of 1-2-3-4 after an idle time of $1.3 \mathrm{~s}$. The signal pattern was repeated continuously until a new haptics code was sent to the MCU. These processes were conducted by programming the MCU.
In the second and third stimulations, we aimed to perform the human-machine cooperation SHM approach by benefiting from both the machine computational process and the human cognitive system to recognize and evaluate the damage for the experiment. As described in the humanmachine cooperative concept section, the formula of 'human + machine' will result in a better process. Here the machine computational process was used to extract the features of the data from the PCA. Once the data were preprocessed, the next step was to encode the data in a form that could be sent to a haptic experimental device. In the second stimulation, the vibrotactile stimuli used in this scheme utilized the encoding of loading patterns obtained from PCA to convey information to human subjects. There are eight different types of vibrotacile value that were extracted from PCA as given in figure 10(a). It is noted that the first and second values were mapped on the first actuator, the third and fourth pattern values mapped on the second actuator, the fifth and sixth values were mapped on the third actuator and the last two values were mapped on the fourth actuator of the glove. When a haptic code threshold was satisfied, the stimulation was displayed to the user with the magnitude of the scaled squared loadings values. The duration for each pattern activation and the pause between them were $1 \mathrm{~s}$ and $0.5 \mathrm{~s}$, respectively. At the end of completion of the fourth vibration motor stimulation, the vibration motors restarted stimulating in an order of 1-2-3-4 after an idle time of $1.3 \mathrm{~s}$. The signal pattern was repeated continuously until a new code was sent to the MCU.

In the third stimulation, all four vibration motors were activated simultaneously to evaluate the subjects' ability to recognize the damage in the tower structure. For instance, the first and the second gray bar values represent the loading scaled scores of the first and second principal components of the first variable, respectively as shown in figure 10(b), and these values were mapped on the first actuator. The activation duration of vibration was $1 \mathrm{~s}$ for each pattern, and the pause between the gray and green bar patterns was $0.5 \mathrm{~s}$. The only difference of the third stimulation from the second stimulation is that all the vibration motors were activated simultaneously in two parts. At the end of activation completion of all vibration motors, they restarted stimulating together after an idle time of $1.3 \mathrm{~s}$. The signal pattern was repeated continuously until a new haptic code was sent to the MCU. It is noted that all the three different stimulation types were conducted with the haptic experimental device by programming the MCU.

\section{Results}

In this study, a new cooperative SHM approach that involves adaptively combining the intelligence of humans with distributed SHM sensing systems was tested. All the tasks explained in methodology were performed on 10 participants, eight males and two females, aged between 25 and 34 years old $(29.6 \pm 4.61)$. They were all right-hand-dominant. Each of the participants was taught which damage scenario was active during the training period. The experiment was performed in 


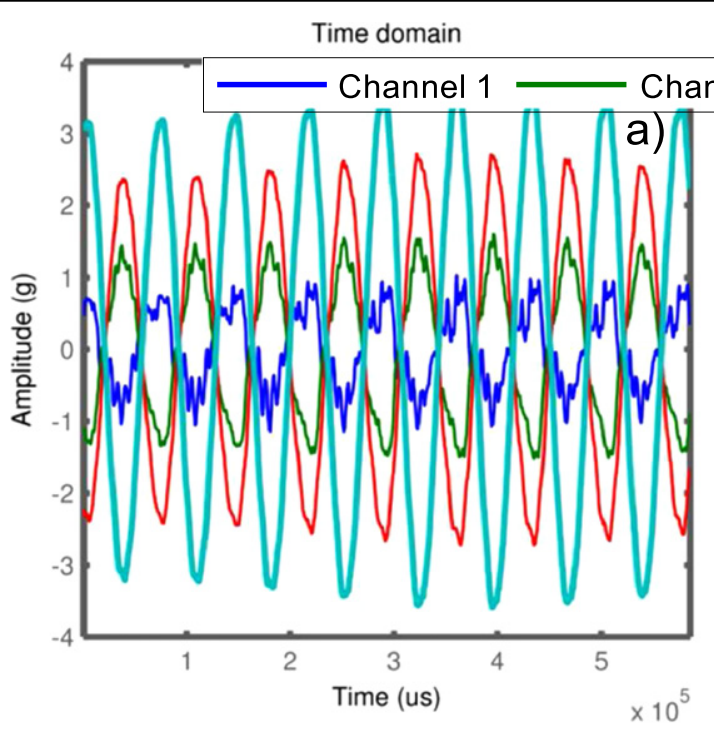

Frequency domain
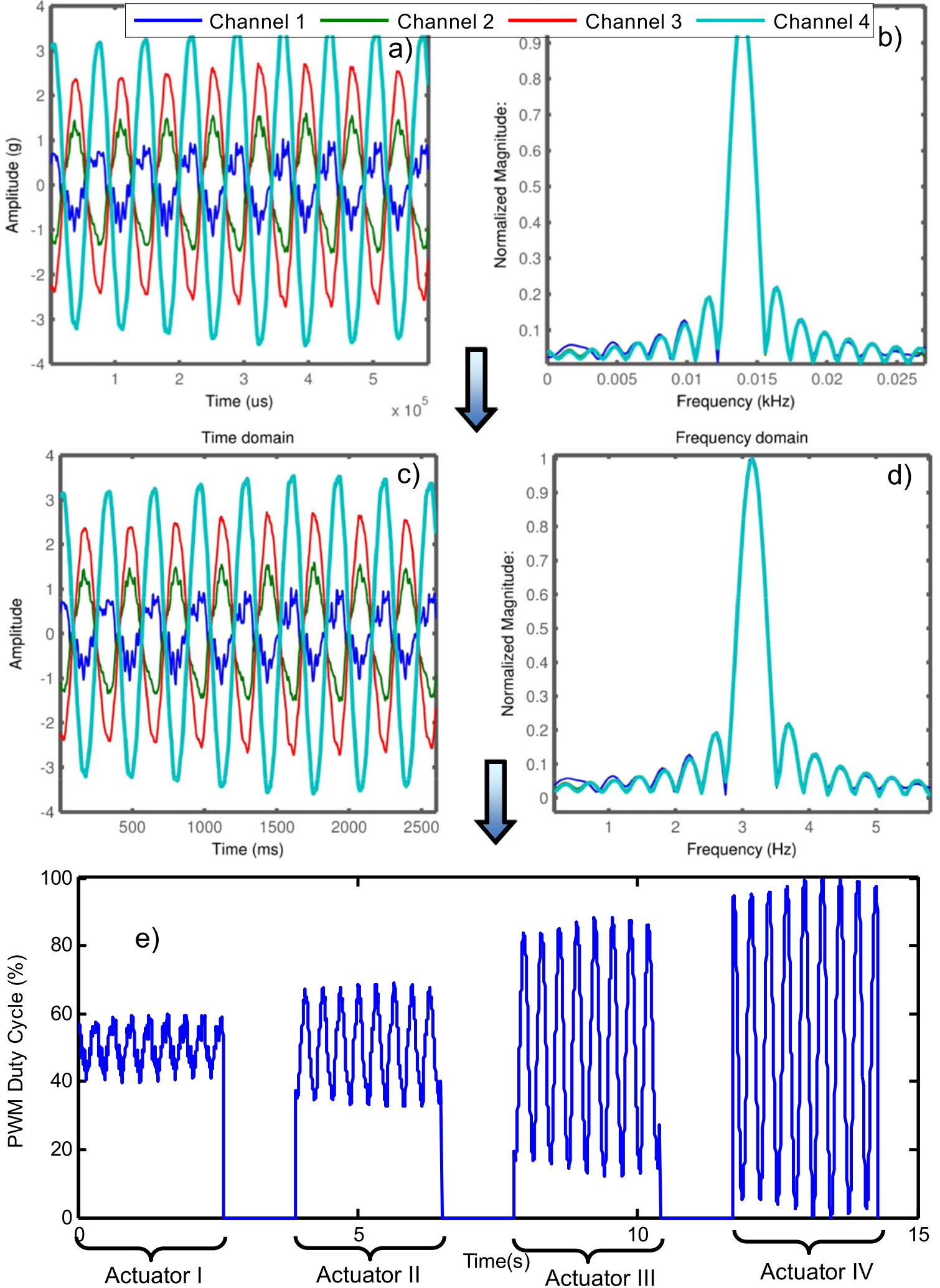

Figure 9. Basic implementation of the first stimulation procedure example for case 1 and test 1. (a) Time points from each signal in a range of 0-585.94 ms are obtained, (b) Frequency domain of these time points. (c) Time signals are modified by reducing the time sampling by a factor of 4.4373; (d) frequency domain of all modified signals. (e) The modified time points were scaled in a range of 0-100 to represent them with the PWM duty cycle. (The duration for each signal shape stimulation and the pause between them was arranged to be $2.6 \mathrm{~s}$ and $1.3 \mathrm{~s}$, respectively.) 


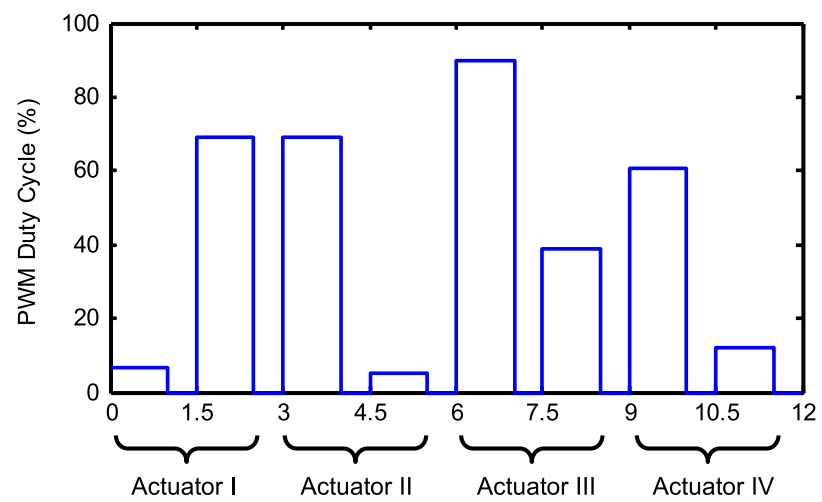

a)

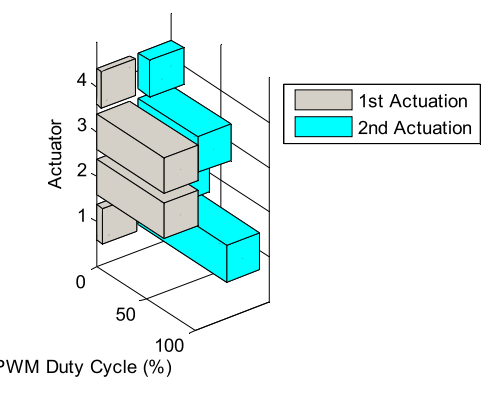

b)

Figure 10. A visual representation of vibrotactile stimuli patterns used in the second and third stimulation by encoding the loading values obtained from the PCA for case 1 and test 1. (a) Stimulation sequence patterns for the second stimuli. The vibrotactile actuators are vibrated in the order of 1-2-3-4. (b) Stimulation sequence patterns for the third stimuli. Here, all the vibrotactile actuators are activated simultaneously.

three sessions. Every session was related to a unique stimuli type. Each of the sessions consisted of 128 trials and 128 tests. 10 min breaks were given at every interval of each session. The participants were instructed to take extra breaks if needed, especially under fatigued or uncomfortable situations. The participants could also abort the experiment at any time if they were was unable to continue due to annoying or uncomfortable reasons. In this experiment, none of the participants aborted the procedure. The experiment task took approximately $120 \mathrm{~min}$ per participant. The assessment was arranged in terms of participants' percentage of correct answers and response times. A correct answer resulted in one point, and an incorrect response resulted in zero points. Then, the maximum number of points was $100 \%$ identification score for the total of each case (correct_response/total number of tests $\times 100)$. All users felt reasonably comfortable wearing the device, and with the seating and hand positioning arrangement. All users felt that they improved their performance during the training period. Some users noted that it required a more conscious effort to improve the percentage of correct answers. The human responses were acquired after completing the test. The participants' results were evaluated statistically with ANOVA to figure out significant differences between human subjects, damage cases, and stimuli type in terms of percentage of correct answers and response time. To determine whether the differences between stimulus means are statistically significant, $p$-value is used to compare the significance level to assess the null hypothesis. Here, $p$-value tests the null hypothesis that data from all groups are drawn from populations with identical means. $P$-values less than 0.05 are accepted as 'statistically significant'.

Results relative to the stimuli types I, II and III are presented in figures 11, 12 and 13, respectively. Here, the $x$ axes denote the case number and $y$ axes denote the human subject number. The intensity of the color scale represents the percentage of correct answers (left-hand side graphs in figures 11-13) and human response time (right-hand side graphs in figures 11-13). In these three stimulating types, no significant differences between human responses were found. However, individual variabilities have been observed as given in figures 11, 12 and 13. These occurred due to the interval variance of mechanoreceptor sensitivity between individuals as well as the types of stimuli presentation. In addition, the individuals' personal motivation to explore stimuli actively is another factor that determined to what degree mechanoreceptors in hands were able to learn, memorize and differentiate the health status of the structure. Additionally, person-related factors that could have played a role in absolute sensation threshold measurements are levels of relaxation and stress, levels of concentration, as well as degree of nervousness.

Figure 11 shows the percentage of correct answers and response times for each of the participants for the first stimuli type. The mean correct percentage of all cases was $18.91 \pm 11.61 \%$ while the mean response time was $12.52 \pm 1.98 \mathrm{~s}$. The mean percentage of highest and lowest correct answers was found in case $6(26.25 \pm 10.94 \%)$ and case $4(10 \pm 7.91 \%)$, while the mean longest and shortest response times based on 10 subjects were observed in case 14 $(13.41 \pm 1.78 \mathrm{~s})$ and case $1(11.61 \pm 0.76 \mathrm{~s})$. The ANOVA test showed that there was no significant difference between the case combinations in terms of percentage of correct answers and response times $(p>0.05)$. As illustrated in figure 11 , the averaged performance for all subjects shows that the individuals could identify structural damage with a low accurate value. In addition, all participants expressed that it was difficult to distinguish between the cases during the training period.

The distribution of percentage of correct answers and response times for each of the participants for the second stimuli type is given in figure 12. In nine participants, the percentages of correct answers were higher than $90 \%$. The mean percentage of highest and lowest correct answers of all humans was found in case $1(95 \pm 12.08 \%)$ and case 10 $(78.75 \pm 32.83 \%)$, while the mean longest and shortest response time was observed in case $3(7.08 \pm 2.7 \mathrm{~s})$ and case $16(4.87 \pm 1.34 \mathrm{~s})$. The averaged percentage of recognizing the right case and answer times of all the human subjects were observed to be $88.13 \pm 20.21 \%$ and $5.87 \pm 2.33$ s, respectively as given in table 2 . One interesting point was that the shortest 


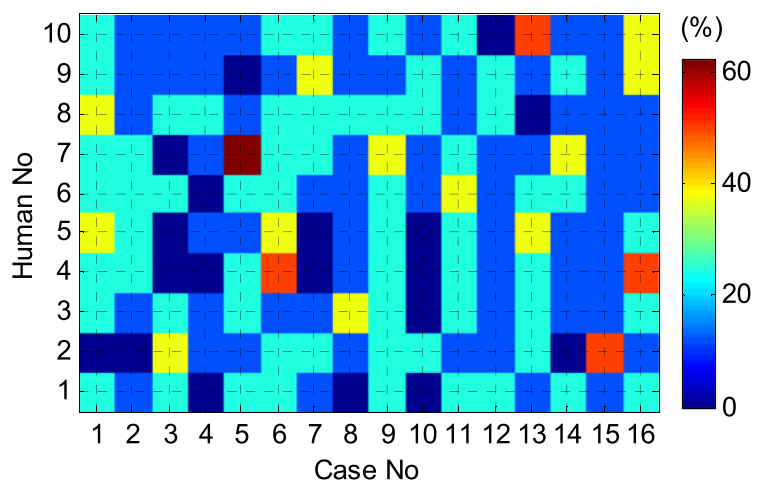

a)

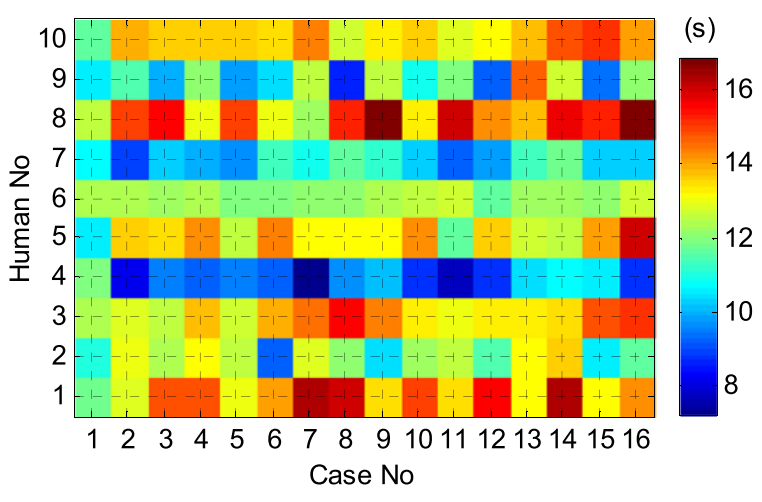

b)

Figure 11. Performance score of all the human subjects for the first stimulus type: (a) percent correct, (b) response time.

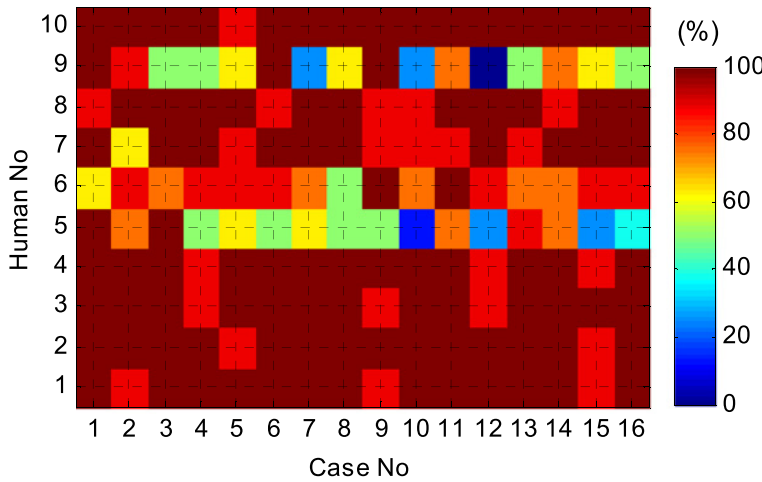

a)

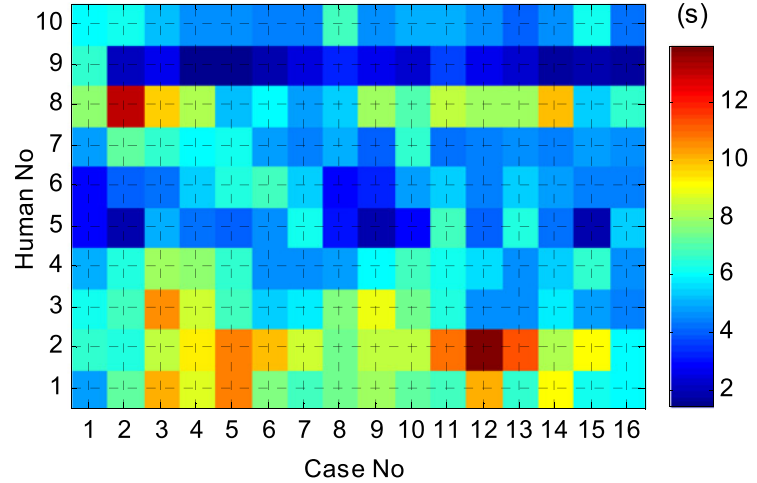

b)

Figure 12. Performance score of all human subjects for the second stimulus type: (a) percent correct, (b) response time.

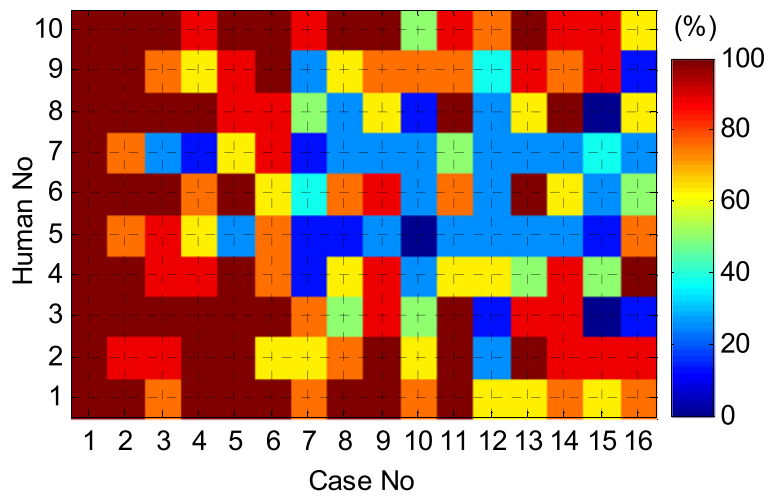

a)

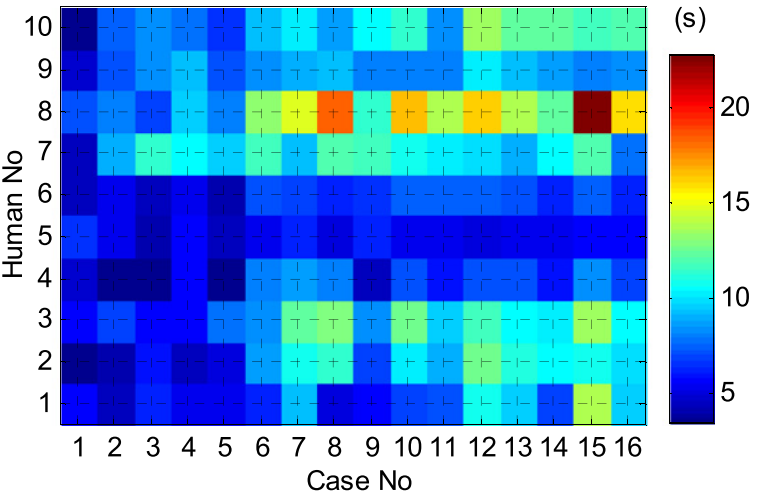

b)

Figure 13. Performance score of all human subjects for the third stimulus type: (a) percent correct, (b) response time.

response time was found in case 16. Figure 12 represents that there was also no direct correlation between the structural case variations in terms of humans' percentage of correct answers in the second type stimulation. There was also no significant difference between cases both in terms of percentage of correct answers $(p=0.916>0.05)$ and response times $(p=0.891>0.05)$. It is noted that all the humans reported that the structural cases could be distinguished better compared to the first one during the training phase. They also reported that they were able to recognize the pattern and trends for all cases easily.
The main objective of the third stimulation was to evaluate the subjects' ability to recognize the damage by activating all of the four vibration motors simultaneously according to results obtained from PCA loading scores. The results obtained from the human subjects for the third stimuli type are given in figure 13 . The mean percentages of the highest and the lowest values of recognizing were found in case $1(100 \%)$ and case $12(37.5 \pm 21.25 \%)$, while the longest and the shortest response times of recognizing the damage were in case $12(11.54 \pm 4.83 \mathrm{~s})$ and case $1(5.10 \pm 1.24 \mathrm{~s})$. The average correct response percentage and response 
Table 2. Statistical results of all human responses for different case and stimulus types.

\begin{tabular}{|c|c|c|c|c|c|c|c|c|}
\hline \multirow[b]{2}{*}{ Case no } & \multicolumn{4}{|c|}{ Percent correct $(\%)$} & \multicolumn{4}{|c|}{ Response time (s) } \\
\hline & $\begin{array}{l}\text { Test stimuli } \\
1(n=10)\end{array}$ & $\begin{array}{l}\text { Test stimuli } \\
2(n=10)\end{array}$ & Test stimuli $3(n=10)$ & $p$ & $\begin{array}{l}\text { Test stimuli } \\
1(n=10)\end{array}$ & $\begin{array}{l}\text { Test stimuli } \\
2(n=10)\end{array}$ & $\begin{array}{l}\text { Test stimuli } \\
3(n=10)\end{array}$ & $p$ \\
\hline 1 & $25.00 \pm 10.21$ & $95.00 \pm 12.08$ & $100.00 \pm 0.00$ & $3.2967 \mathrm{E}-17$ & $11.61 \pm 0.76$ & $5.40 \pm 1.60$ & $5.10 \pm 1.24$ & $1.8073 \mathrm{E}-12$ \\
\hline 2 & $16.25 \pm 8.44$ & $90.00 \pm 12.91$ & $93.75 \pm 10.62$ & $8.2377 \mathrm{E}-16$ & $12.27 \pm 2.14$ & $6.13 \pm 3.15$ & $6.25 \pm 1.86$ & 0.000004 \\
\hline 3 & $16.25 \pm 13.24$ & $92.50 \pm 16.87$ & $83.75 \pm 22.86$ & $4.3408 \mathrm{E}-10$ & $12.48 \pm 2.02$ & $7.08 \pm 2.70$ & $6.62 \pm 2.40$ & 0.000009 \\
\hline 4 & $10.00 \pm 7.91$ & $86.25 \pm 19.94$ & $78.75 \pm 27.67$ & $3.8303 \mathrm{E}-9$ & $12.66 \pm 1.78$ & $6.49 \pm 2.50$ & $7.01 \pm 2.10$ & $6.2356 \mathrm{E}-7$ \\
\hline 5 & $21.25 \pm 16.72$ & $87.50 \pm 14.43$ & $86.25 \pm 24.62$ & $9.8727 \mathrm{E}-9$ & $12.07 \pm 1.83$ & $6.34 \pm 2.81$ & $6.23 \pm 1.95$ & 0.000002 \\
\hline 6 & $26.25 \pm 10.94$ & $92.50 \pm 15.81$ & $85.00 \pm 15.37$ & $4.6384 \mathrm{E}-11$ & $12.13 \pm 1.98$ & $5.65 \pm 2.14$ & $8.76 \pm 2.33$ & 0.000002 \\
\hline 7 & $17.50 \pm 12.08$ & $86.25 \pm 25.31$ & $45.00 \pm 28.99$ & 0.000002 & $12.64 \pm 2.41$ & $5.39 \pm 1.60$ & $9.82 \pm 2.50$ & $3.2201 \mathrm{E}-7$ \\
\hline 8 & $15.00 \pm 9.86$ & $86.25 \pm 22.40$ & $58.75 \pm 30.65$ & $6.7325 \mathrm{E}-7$ & $12.73 \pm 2.41$ & $5.38 \pm 1.89$ & $9.82 \pm 4.11$ & 0.000031 \\
\hline 9 & $25.00 \pm 5.89$ & $90.00 \pm 15.37$ & $75.00 \pm 28.87$ & $8.8069 \mathrm{E}-8$ & $12.82 \pm 1.97$ & $5.60 \pm 2.61$ & $8.06 \pm 2.53$ & 0.000001 \\
\hline 10 & $11.25 \pm 10.94$ & $78.75 \pm 32.83$ & $40.00 \pm 26.22$ & 0.000010 & $12.44 \pm 1.90$ & $5.86 \pm 2.00$ & $9.75 \pm 3.34$ & 0.000013 \\
\hline 11 & $22.50 \pm 7.91$ & $93.75 \pm 10.62$ & $77.50 \pm 25.55$ & $7.5026 \mathrm{E}-10$ & $12.16 \pm 2.30$ & $6.44 \pm 2.08$ & $8.55 \pm 2.39$ & 0.000022 \\
\hline 12 & $15.00 \pm 7.91$ & $78.75 \pm 35.87$ & $37.50 \pm 21.25$ & 0.000014 & $12.10 \pm 2.27$ & $6.29 \pm 3.44$ & $10.57 \pm 3.33$ & 0.000680 \\
\hline 13 & $22.50 \pm 14.19$ & $90.00 \pm 16.46$ & $70.00 \pm 29.58$ & $3.9133 \mathrm{E}-7$ & $12.93 \pm 1.25$ & $5.83 \pm 2.45$ & $9.65 \pm 2.60$ & $4.2772 \mathrm{E}-7$ \\
\hline 14 & $17.50 \pm 10.54$ & $91.25 \pm 11.86$ & $71.25 \pm 26.39$ & $1.9941 \mathrm{E}-9$ & $13.41 \pm 1.78$ & $5.86 \pm 2.51$ & $9.00 \pm 2.64$ & $4.5636 \mathrm{E}-7$ \\
\hline 15 & $16.25 \pm 11.86$ & $83.75 \pm 23.61$ & $45.00 \pm 35.45$ & 0.000013 & $12.59 \pm 2.25$ & $5.24 \pm 2.15$ & $11.55 \pm 4.83$ & 0.000057 \\
\hline 16 & $25.00 \pm 13.18$ & $87.50 \pm 23.57$ & $56.25 \pm 30.76$ & 0.000013 & $13.21 \pm 2.56$ & $4.87 \pm 1.34$ & $9.44 \pm 3.08$ & $1.6573 \mathrm{E}-7$ \\
\hline Total $(n=160)$ & $18.91 \pm 11.64$ & $88.13 \pm 20.21$ & $68.98 \pm 30.91$ & $3.47 \mathrm{E}-104$ & $12.52 \pm 1.98$ & $5.87 \pm 2.33$ & $8.51 \pm 3.22$ & 7.377E-80 \\
\hline
\end{tabular}




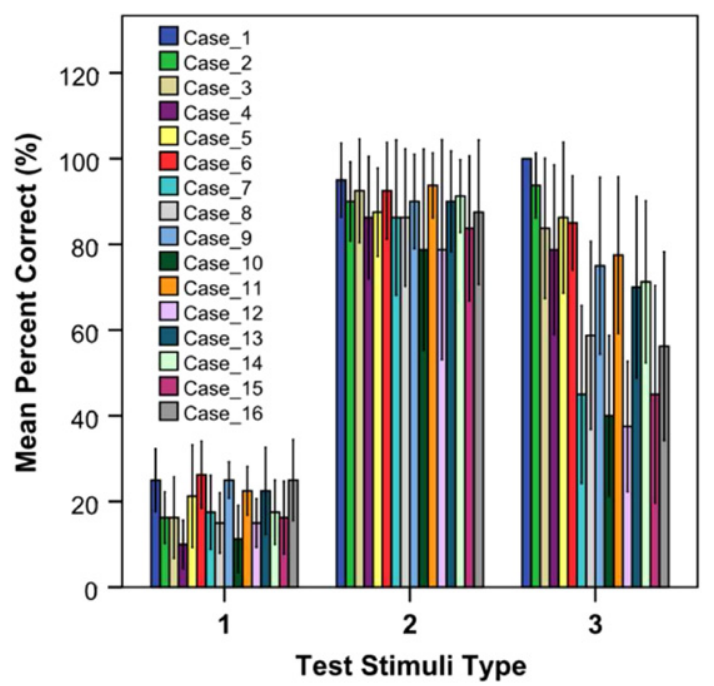

a)

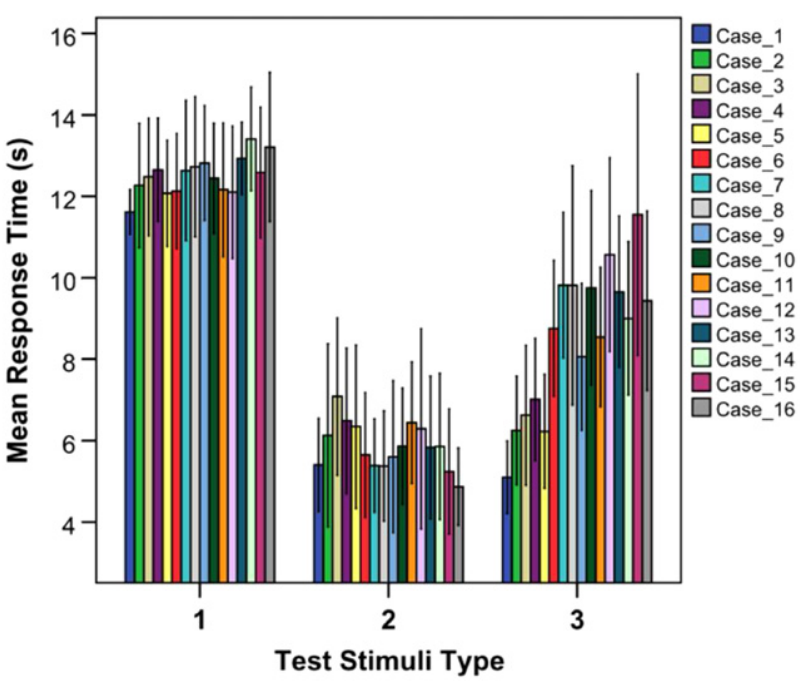

b)

Figure 14. Summary of human subject results in all cases in terms of (a) percent correct, (b) response time. (Error bars: $95 \%$ confidence interval.)

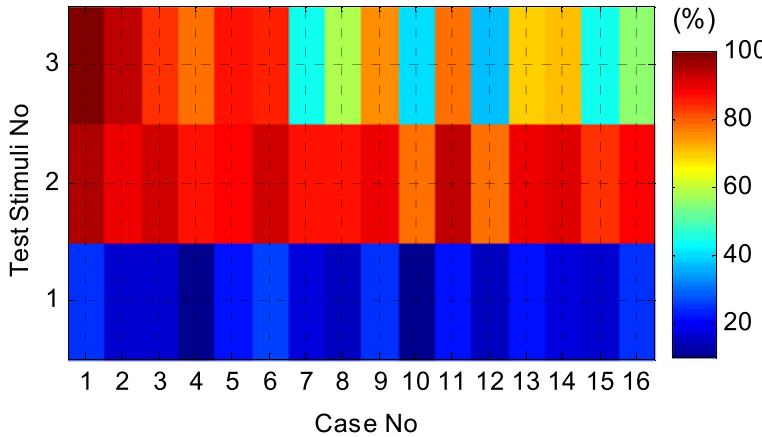

a)

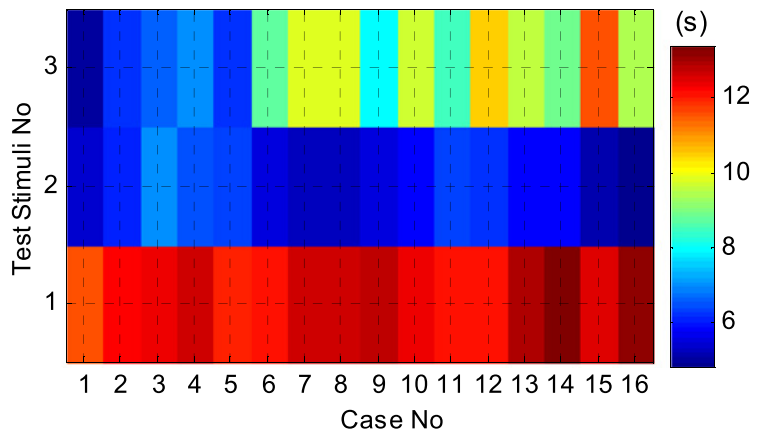

b)

Figure 15. Contour plots of all human subjects for all cases: (a) percent correct, (b) response time.

duration in test stimuli session III were equal to $68.98 \pm 30.91 \%$ and $8.51 \pm 3.22 \mathrm{~s}$, respectively, based on the result given in table 2 . The ANOVA results demonstrated that there was a significant difference between cases in terms of percentage of correct response $(p<0.001)$ and response time $(p<0.001)$.

Figure 14 shows the bar graphs of the aforementioned averaged values and standard error of three different stimuli of the 10 humans for 16 cases. As discussed before, the upper and lower percentages of the humans' correct answers show that the structural cases were not significantly different from each other in test stimuli I and II, but were significantly different for test stimuli III. This result probably reflects that the human subjects had no difficulty in recognizing and discriminating the appropriate damage combinations in test stimuli II.

The results obtained from human subjects for three different stimulations are also illustrated in contour plots. The $x$ and $y$ axes given in figure 15 represent the case number as identified in table 1 and test stimuli number, respectively. In the data analysis, values of human correct responses as well as their response times for three different stimulus types were analyzed in order to seek an effective stimulus type for SHM applications. The averaged percentages of correct answers were $18.91 \pm 11 \%, 88.13 \pm 20.21 \%$ and $68.98 \pm 30.91 \%$, respectively and the averaged response times were $12.52 \pm 1.98 \%, 5.87 \pm 2.33 \%, 8.51 \pm 3.22 \%$ for the first, second and third stimuli types as given in figure 16 and table 2 .

An assessment was performed to determine the robustness of test stimuli types in terms of percentage correct answer and response time of humans via the Tukey test as given in tables 3 and 4 respectively. Significant differences between the types of stimuli were found during the statistical analysis. The results for test stimuli I were significantly lower than test stimuli II $(p<0.01)$ and test stimuli III $(p<0.01)$ in terms of percentage of correct responses, but they were higher than stimuli II and test stimuli III in terms of response time. While the human results in test stimuli II were significantly higher than test stimuli I $(p<0.01)$ and test stimuli III $(p<0.01)$ in terms of percentage of correct responses, they were lower than I $(p<0.01)$ and III $(p<0.01)$ in terms of response time. Finally, test stimuli III had significantly higher values of correct answer percentage than test stimuli I $(p<0.01)$, but lower than test stimuli II $(p<0.01)$ as well as 


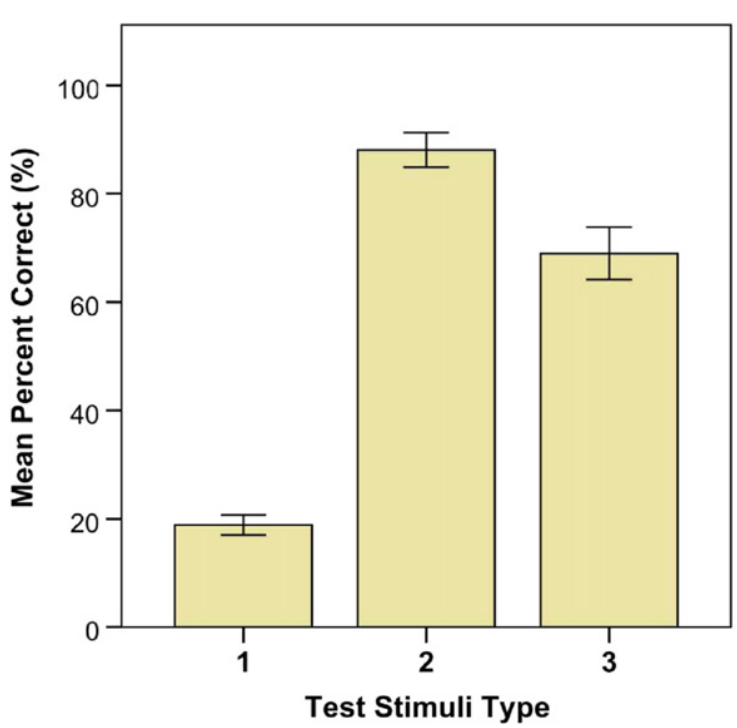

a)

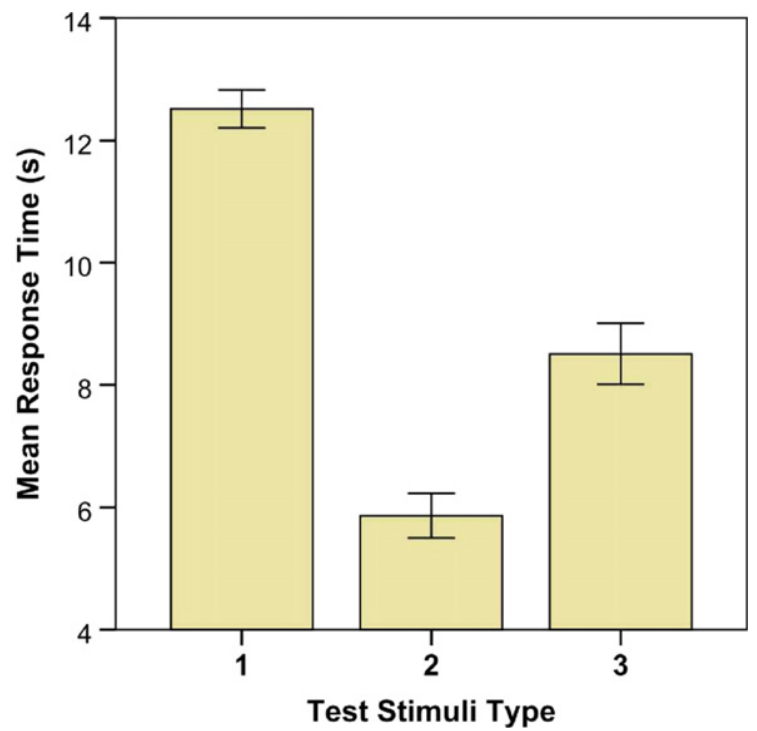

b)

Figure 16. All human subject results for different test stimuli for all cases: (a) correct percent, (b) human response time. (Error bars: 95\% confidence interval.)

Table 3. Multiple comparison of the human percent correct results with the Tukey test for all cases.

\begin{tabular}{llcrlrrr}
\hline & & & & & \multicolumn{2}{c}{$95 \%$ Confidence interval } \\
\cline { 5 - 7 }$(I)$ Test_stimuli & $(J)$ Test_stimuli & Mean difference $(I-J)$ & Std. error & $p^{\text {a }}$ & Lower bound & Upper bound \\
\hline 1 & 2 & -69.21875 & 2.49928 & $5.1 \mathrm{E}-9$ & -75.0947 & -63.3428 \\
& 3 & -50.07813 & 2.49928 & $5.1 \mathrm{E}-9$ & -55.9541 & -44.2022 \\
2 & 1 & 69.21875 & 2.49928 & $5.1 \mathrm{E}-9$ & 63.3428 & 75.0947 \\
& 3 & 19.14063 & 2.49928 & $5.1 \mathrm{E}-9$ & 13.2647 & 25.0166 \\
& 1 & 50.07813 & 2.49928 & $5.1 \mathrm{E}-9$ & 44.2022 & 55.9541 \\
& 2 & -19.14063 & 2.49928 & $5.1 \mathrm{E}-9$ & -25.0166 & -13.2647 \\
\hline
\end{tabular}

Significant at the 0.05 level.

Table 4. Multiple comparison of the human response time results with the Tukey test for all cases.

\begin{tabular}{llcrrrr}
\hline & & & & & \multicolumn{2}{c}{$95 \%$ Confidence interval } \\
\cline { 5 - 7 }$(I)$ Test_stimuli & $(J)$ Test_stimuli & Mean difference $(I-J)$ & Std. error & $p^{\text {a }}$ & Lower bound & Upper bound \\
\hline 1 & 2 & 6.64991 & .28630 & $5.1002 \mathrm{E}-9$ & 5.9768 & 7.3230 \\
2 & 3 & 4.00499 & .28630 & $5.1002 \mathrm{E}-9$ & 3.3319 & 4.6781 \\
& 1 & -6.64991 & .28630 & $5.1002 \mathrm{E}-9$ & -7.3230 & -5.9768 \\
3 & 3 & -2.64492 & .28630 & $5.1002 \mathrm{E}-9$ & -3.3180 & -1.9718 \\
& 1 & -4.00499 & .28630 & $5.1002 \mathrm{E}-9$ & -4.6781 & -3.3319 \\
& 2 & 2.64492 & .28630 & $5.1002 \mathrm{E}-9$ & 1.9718 & 3.3180 \\
\hline
\end{tabular}

a Significant at the 0.05 level.

lower human response durations than test stimuli I $(p<0.01)$ and higher than test stimuli II $(p<0.01)$, (tables 3 and 4$)$.

\section{Discussion and conclusion}

This study demonstrated a new, simple and noninvasive human-machine cooperation approach for SHM applications. The inspiration comes from the historical advances in the neuroscience community. The neuroscience field has investigated haptic-based methods for replacing lost sensations. In this study, we investigated the probability of delivering humans a sense that represents the health of the structure. Here, the collaboration system is composed of both computations of machines and the human cognitive system.

First, a steel tower structure having bolted lattices was manufactured as a test bed, which could be set to show non-linear dynamic responses by loosening the bolts. Then, signal processing was used that takes four acceleration time series measurements, then reduces the 


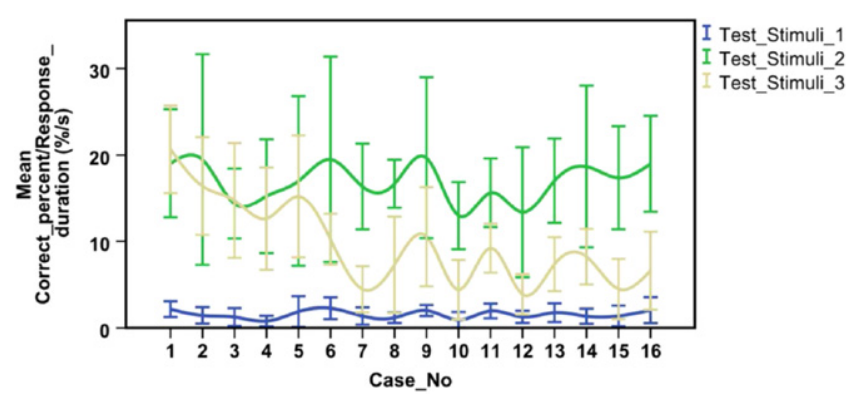

Figure 17. Proportion values of CorrectPercent to ResponseTime of the three different test stimuli for all cases. (Error bars: standard deviation.)

dimensionality of those data by using PCA. PCA is commonly used for dimensional reduction of data for the preprocessing step when training using the ANN (Figueiredo et al 2011). In this study, the ANN was replaced with the human NN by using vibrotactile elements. To investigate the improved approach to damage detection, three different stimulation types were designed and each of them was tested independently on ten human subjects. The first stimulation type is based on the encoding of the waveform of the acceleration measurement for humans to find patterns in SHM. The test stimuli I experiment showed that subjects were able to discriminate the current structural health with a low value (success $<19 \%$ ). Since no statistically significant differences in discrimination percentage across the 16 cases were found, it can be concluded that the capability of subjects to detect the right case did not change. In addition to that, it was found that the subjects did not have a high capability to localize the damage with acceleration waveforms for this experiment. The test stimuli II experiment was designed as a concept-proof of the cooperative human-machine SHM approach. The most promising results obtained in this experiment showed that the subjects were able to localize the damage with a high performance (success $<88 \%$ ).

Application of the concept to the tower structure demonstrated that the developed cooperative approach is effective in discriminating the corresponding combinational values of various types of bolt-loosened damage. In test stimuli II, although the outcomes demonstrated low success in terms of correct answers per time for the first case, it was found to be higher than in some other cases as given in figure 17. The test stimuli III experiment showed that the subjects were able to localize the damage with a mean percentage of $69 \%$. Here, the highest and lowest values of CorrectPercent/ResponseTime were found in human subject 2 $\left(12.98 \pm 5.55 \% \mathrm{~s}^{-1}\right)$ and human subject $5\left(7.61 \pm 5.94 \% \mathrm{~s}^{-1}\right)$, respectively. In addition, the highest and lowest values of CorrectPercent/ResponseTime were found in human case 1 $\left(19.81 \pm 5.33 \% \mathrm{~s}^{-1}\right)$ and case $2\left(17.93 \pm 9.14 \% \mathrm{~s}^{-1}\right)$, respectively. The trend of CorrectPercent/ResponseTime decreased until case 7 for the third stimulation. This result probably shows that the greater the complexity and damage in structures, the worse the discrimination being achieved. This result is in accordance with the psychophysical haptics study of
Ciprinai et al (2012). However, this trend was found to be opposite for cases 5, 9, 11 and 14. The human subjects' results showed that sorting the three different stimuli types in terms of correct answer per time from high to low is test stimuli II, I and III.

The limitation of this study is that the structural tower was tested for a structure excited at sinusoidal signals in well controlled laboratory settings. However, real world engineering structures are subjected to environmental and operational variations. Sometimes these variations can mask the damage related to the features of correlation between the magnitude of these features and damage level. The obtained results are encouraging, but further investigations will be needed to test the validity of the technique with other data related to environmental and operational conditions before it can be used in real engineering structures.

In this study, three different vibrotactile stimulations were used. However, what type of stimulation and how much information should be provided are still open questions, and still impossible to tackle without a practical validation. In others words, it should be noted that it is not possible to conduct experiments requiring thousands of trials which may take days for participants to complete. However, in the authors' opinion, for enriched recognition of the patterns of the damage, three types of basic information need to be considered when improving a stimulation in future studies: (i) vibrotactile coding type of the SHM measurement data, (ii) vibrotactile map pattern (where the tactile actuation is taking place, which part of the human should be stimulated) and (iii) vibrotactile frequency and amplitude.

In conclusion, as an alternative to other SHM techniques, a human-machine cooperation approach concept was tested on time series data obtained from the structural tower to a build a highly computational performance of human and machine teaming with the idea of sensory substitution. The results showed that this technique appears to be a promising tool for SHM applications. To further verify the technique, future efforts should be directed to applicability of this technique in various structures including real-world applications with large sensor networks.

\section{Acknowledgments}

The authors would like to thank all the volunteers who took part in the experimental tests. This study was partially supported by Ege University, Office of Scientific Research Projects (12-MÜH-046). Mahmut Pekedis was also supported by the Council of higher education of Turkey in Los Alamos National Laboratory, 2012-2013. The human subjects' tests were conducted under the directions of Ege University, Clinical Research Ethical Board, with a document number of $13-11 / 104$ in 2013. 


\section{References}

Bach-y-Rita P and Stephen W K 2003 Sensory substitution and the human-machine interface Trends Cogn. Syst. 7 541-6

Bainbridge L 1987 Ironies of automation New Technology and Human Error ed J Rasmussen et al (Chichester: Wiley) pp 271-84

Balageas D, Fritzen C P and Güemes A 2006 Structural Health Monitoring (London: ISTE Ltd)

Barlow H B 1969 Pattern recognition and the responses of sensory neurons Ann. New York Acad. Sci. $156872-81$

Bear M, Connors B and Paradiso M A 2007 Neuroscience: Exploring the Brain (Philadelphia, PA: Lippincott Williams and Wilkins)

Beral B and Speckmann H 2003 Structure health monitoring (SHM) for aircraft structures: a challenge for system developers and aircraft manufacturers, structural health monitoring, from diagnostics and prognostics to structural health management Proc. 4th Int. Workshop on Structural Health Monitoring (Stanford, CA) (Lancaster, PA: Destech Publications Inc.) pp 12-29

Block J, Djidjev S, Jameson N and Mascarenas D 2014 Comparing statistical classification with a vibro-tactile human-machine interface for structural health monitoring Conf. Proc. Soc. for Experimental Mechanics Series, Dynamics of Civil Structures vol 4, pp 441-9

Brown C, Cowell M, Plont C A, Hahn H and Mascareñas D A 2013 Vibro-haptic human-machine interface for structural health monitoring applications Conf. Proc. Society for Experimental Mechanics Series, Special Topics in Structural Dynamics vol 6, pp 187-97

Böger L, Wichmann M H G, Meyer L O and Schulte K 2008 Load and health monitoring in glass fibre reinforced composites with an electrically conductive nanocomposite epoxy matrix Compos. Sci. Technol. 68 1886-94

Chan A, MacLean K and McGrenere J 2005 Learning and identifying haptic icons under workload Proc. World Haptics, Pisa pp 432-9

Cheung A, Cabrera C, Sarabandi P, KNair K, Kiremidjian A and Wenzel H 2008 The application of statistical pattern recognition methods for damage detection to field data Smart Mater. Struct. 17065023

Christian P, Artem K and Stefan S 2006 Design and evaluation of low cost force feedback system for myoelectric prosthetic hands J. Prosthet. Orthot. 18 57-61

Cipriani C, Alonzo M D and Carrozza C 2012 A miniature vibrotactile sensory substitution device for multifingered hand prosthetics IEEE Trans. Biomed. Eng. 59 400-8

Dashti H T et al 2010 Pattern recognition in collective cognitive systems: hybrid human-machine learning (HHML) by heterogeneous ensemble arXiv:1008.5387

Dhillon G S and Horch K W 2005 Direct neural sensory feedback and control of prosthetics arm IEEE Trans. Neural Syst. Rehabil. Eng. 13 468-72

Enriquez M and MacLean K 2008 The role of choice in longitudinal recall of meaningful tactile signals Proc. Symp. Haptic Interfaces for Virtual Environments and Teleoperator Systems pp 49-56

Farrar C R and Worden K 2013 Structural Health Monitoring a Machine Learning Perspective (Chichester: Wiley)

Figueiredo E, Park G, Farrar C R, Worden K and Figueiras J 2011 Machine learning algorithms for damage detection under operational and environmental variability Struct. Health Monit. 10 559-72

Gescheider G A 1997 Psychophysics: The Fundamentals 3rd edn (Mahwah, NJ: Lawrence Erlbaum Associates)
Grosse-Wentrup M, Mattia D and Oweiss K 2011 Using braincomputer interfaces to induce neural plasticity and restore function J. Neural. Eng. 18025004

Hoc J M 2000 From human machine interaction to human machine cooperatio Ergonomics 43833843

Loh K J and Azhari F 2012 Recent advances in skin-inspired sensors enabled by nanotechnology J. Mater. 64 793-801

Loh K J, Lynch J P, Shim B S and Kotov N 2008 Tailoring piezoresistive sensitivity of multilayer carbon nanotube composite strain sensors J. Intell. Mater. Syst. Struct. 19 747-64

Loh K J, Ryu D and Lee B M 2015 Bio-inspired sensors for structural health monitoring Biotechnologies and Biomimetics for Civil Engineering (Cham: Springer International)

Loyola B R, la Saponara V and Loh K J 2010 In situ strain monitoring of fiber-reinforced polymers using embedded piezoresistive nanocomposites J. Mater. Sci. 45 6786-98

Mahns D A, Perkins M, Sahai V, Robinson L and Rowe M J 2006 Vibrotactile frequency discrimination in human hairy skin J. Neurophysiol. 95 1442-50

Mansfield N J 2005 Human Response to Vibration (Boca Raton, FL: Taylor and Francis e-Library)

Mascarenas D, Choi Y, Kim H C, Pekedis M, Lee J R, Farrar C and Hong S C 2013 Development of a novel human-machine interface exploiting sensor substitution for structural health monitoring 2013 IEEE RO-MAN: The 22nd IEEE Int. Symp. Robot and Human Interactive Communication (Gyeongju, South Korea) pp 334-5

Mascareñas D, Plont C A, Brown C, Cowell M, Jameson N J, Block J, Djidjev S, Hahn H and Farrar C A 2014 A vibrohaptic human-machine interface for structural health monitoring Struct. Health Monit. 13 671-85

Mascarenas D et al 2013 A vibro-haptic human-machine interface for structural health monitoring The 9th Int. Workshop on Structural Health Monitoring (Structural Health Monitoring 2013: A Roadmap to Intelligent Structures vol 1) (September 10-12, Stanford, CA) (Destech Publications Inc) pp 1171-8

Melchner L V, Pallas S and Sur M 2000 Visual behavior mediated by retinal projections directed to the auditory pathway Nature $404871-6$

Merzenich M M and Harrington T 1969 The sense of fluttervibration evoked by stimulation of the hairy skin of primates: comparison of human sensory capacity with responses of mechanoreceptive afferents innervating the hairy skin of monkeys Exp. Brain Res. 9 236-60

Morley J W and Rowe M J 1990 Perceived pitch of vibrotactile stimuli: effects of vibration amplitude, and implications for vibration frequency coding J. Physiol. 431 403-16

Myle K and Binseel M 2007 The tactile modality: a review of tactile sensitivity and human tactile interfaces Army Research Laboratory, Report No : ARL-TR-4115

Nofar M, Hoa S V and Pugh M D 2009 Development of novel single-wall carbon nanotube-epoxy composite ply actuators Compos. Sci. Technol. 69 1599-606

Pearson K 1901 On lines and planes of closest fit to systems of points in space Phil. Mag. 2 559-72

Salowitz N, Guo Z, Li Y-H, Kim K, Lanzara G and Chang F-K 2013 Bio-inspired stretchable network-based intelligent composites J. Compos. Mater. 47 97-105

Schmidt R F 1986 Fundamentals of Sensory Physiology (New York: Springer)

Schwamb M E et al 2012 A transiting circumbinary planet in a quadriple star system arXiv:1210.3612

Sharma J, Angelucci A and Sur M 2000 Induction of visual orientation modules in auditory cortex Nature $404841-7$

Sohn H and Farrar C R 2001 Damage diagnosis using time series analysis of vibration signals Smart Mater. Struct. 10 446-51 
Stallkamp J, Schlipsing M, Salmen J and Igel C 2012 Man versus computer: benchmarking machine learning algorithms for traffic sign recognition Neural Netw. 32 323-32

Stoffren T A and Pittenger J B 1995 Human echolocation as basic form of perception and action Ecol. Psychol. 7 181-215

Vapnik V N 1998 The Nature of Statistical Learning Theory (New York: Springer)

Verrillo R T 1985 Psychophysics of vibrotactile stimulation $J$. Acoust. Soc. Am. 77 225-32

Verrillo R T, Fraioli A J and Smith R L 1969 Sensation magnitude of vibrotactile stimuli Attention Perception Psychophys. 6 366-73
Visell Y 2009 Tactile sensory substitution: models for enaction in HCI Interact. Comput. 21 38-53

Vonderhaegen F, Crevits I, Debernard S and Millot P 1994 Human machine cooperation: toward and activity regulation assistance for different air-traffic control levels Int. J. Hum.-Comput. Interact. 6 65-104

Weiskroniz L 1986 Blindsight: A Case Study and Implications (Oxford: Clarendon)

Zhu D, Yi X, Wang Y, Lee K-M and Guo J 2010 A mobile sensing system for structural health monitoring: design and validation Smart Mater. Struct. 19055011 